This item was submitted to Loughborough's Research Repository by the author.

Items in Figshare are protected by copyright, with all rights reserved, unless otherwise indicated.

\title{
Validity of the weakly nonlinear solution of the cauchy problem for the boussinesq-type equation
}

PLEASE CITE THE PUBLISHED VERSION

http://dx.doi.org/10.1111/sapm.12034

\section{PUBLISHER}

(c) Wiley

VERSION

AM (Accepted Manuscript)

\section{PUBLISHER STATEMENT}

This work is made available according to the conditions of the Creative Commons Attribution-NonCommercialNoDerivatives 4.0 International (CC BY-NC-ND 4.0) licence. Full details of this licence are available at: https://creativecommons.org/licenses/by-nc-nd/4.0/

\section{LICENCE}

CC BY-NC-ND 4.0

\section{REPOSITORY RECORD}

Khusnutdinova, Karima R., Kieron R. Moore, and Dmitry Pelinovsky. 2019. "Validity of the Weakly Nonlinear Solution of the Cauchy Problem for the Boussinesq-type Equation". figshare.

https://hdl.handle.net/2134/20162. 


\title{
VALIDITY OF THE WEAKLY-NONLINEAR SOLUTION OF THE CAUCHY PROBLEM FOR THE BOUSSINESQ-TYPE EQUATION
}

\author{
K.R. KHUSNUTDINOVA ${ }^{1}$, K.R. MOORE ${ }^{1}$, AND D.E. PELINOVSKY ${ }^{2}$
}

\begin{abstract}
We consider the initial-value problem for the regularized Boussinesq-type equation in the class of periodic functions. Validity of the weakly-nonlinear solution, given in terms of two counter-propagating waves satisfying the uncoupled Ostrovsky equations, is examined. We prove analytically and illustrate numerically that the improved accuracy of the solution can be achieved at the time scales of the Ostrovsky equation if solutions of the linearized Ostrovsky equations are incorporated into the asymptotic solution. Compared to the previous literature, we show that the approximation error can be controlled in the energy space of periodic functions and the nonzero mean values of the periodic functions can be naturally incorporated in the justification analysis.
\end{abstract}

\section{INTRODUCTION}

Validity of the long-wave approximation for shallow water waves has been considered in many recent works. Unbounded spatial domains and classes of decaying initial data were typically considered. The first results in this direction were found in the context of water waves by Craig [10], Schneider [36], Schneider \& Wayne [37, 38], Ben Youssef \& Colin [1], and Lannes [26]. Rigorous justification analysis was developed to control the approximation error, and the bounds on the error terms were typically found to be larger than those in the formal asymptotic theory.

Wayne \& Wright [42] extended this analysis to the regularized Boussinesq equation to incorporate the first-order correction to the leading-order approximation and to control the error term in Sobolev spaces (see [43] for a similar treatment of the original water wave equations). They also reported numerical approximations that illustrated the validity of the main result, where the bounds on the approximation error were controlled to be of the same size as in the formal asymptotic theory.

Comprehensive treatment of the Boussinesq systems was developed by Bona et al. [7], who explored the case of symmetric Boussinesq systems and justified the long-wave approximation (where the first-order correction term was added to the leading-order term) in a number of physical models that included the water-wave equations and the regularized Boussinesq system. A priori energy estimates and Gronwall's inequalities were used to control the error term in Sobolev spaces and to recover the error bounds of the formal asymptotic theory. Initial data on the infinite line with sufficient decay at infinity were treated on equal footing with the periodic initial data under the zero mean-value assumption. The approximation error for the periodic data with a nonzero mean value was found to be significantly larger.

Date: November 7, 2013. 
Recently, in the framework of a system of coupled Boussinesq equations [18], the long-wave approximation was extended to the regularized Boussinesq-type equation

$$
u_{\tau \tau}-u_{\xi \xi}+\delta u=\frac{1}{2}\left(u^{2}\right)_{\xi \xi}+u_{\tau \tau \xi \xi},
$$

where $\delta>0$ and the subscripts denote partial differentiation. Equation (1) with $\delta>0$ arises also in the context of oceanic waves, which takes into account the effect of background rotation [13. Therefore it is sometimes called the rotation modified Boussinesq equation. This equation is a two-directional version of the Ostrovsky equation [29], which constitutes a modification of the Korteweg-de Vries (KdV) equation with an additional term for $\delta \neq 0$. More recently, regularized Boussinesq-type equations with $\delta>0$ have appeared in the context of a modified Toda lattice on an elastic substrate 44] and a layered solid waveguide with the soft bonding layer [20, 21]. For brevity, we will call the equation (1) the regularized Boussinesq equation regardless of the value of $\delta$.

We shall note in passing that, in the water wave context, the accuracy of the equation (1) does not exceed the accuracy of the KdV or Ostrovsky equation, since the one-way propagation is assumed in order to derive this equation from the Boussinesq system. However, we would like to emphasize that equation (1) with both $\delta=0$ and $\delta>0$ is a valid twodirectional model in the context of the waves in various solid waveguides (see, for example, [34, 35] and [20, 21, 44]). In particular, equation (1) with $\delta>0$ constitutes a reduction of the system of coupled regularised Boussinesq equations describing waves in layered elastic waveguides with the soft bonding layer (see [21]) in the limit when the bonding coefficient in one of the layers is much smaller than in the other.

A formal weakly-nonlinear solution of the initial-value problem for a system of coupled regularized Boussinesq equations on the infinite interval has been constructed in terms of solutions of coupled and uncoupled Ostrovsky equations for unidirectional counter-propagating waves in the recent work [18]. Numerical simulations showed generation of a radiating solitary wave (in the case of strong interactions) and strongly nonlinear wave packets (in the case of weak interactions) from localized initial data. Radiating solitary waves in coupled $\mathrm{KdV}$ equations were previously observed in [12]. The emergence of the strongly nonlinear wave packets for the Ostrovsky equation was reported in [14. A discrete version of the same phenomenon was studied in [44]. The weak interaction scenario leading to two uncoupled Ostrovsky equations remains valid in the case of the scalar regularized Boussinesq equation (1) with $\delta>0$.

Explicit analytical solutions and more detailed numerical simulations were developed in the follow-up work [19] in the context of the regularized Boussinesq equation with $\delta=0$. In particular, the explicit weakly nonlinear solution of the regularized Boussinesq equation was constructed for the initial data corresponding to the soliton solution of the KdV equation. The solution shows generation of a small counter-propagating solitary wave, which agrees with the numerical simulations. Explicit analytical solutions have also been constructed for the initial data in the form of the $N$-soliton solutions of the $\mathrm{KdV}$ equation and their perturbations. It was shown in [19] that the error term is significantly smaller when the first-order correction term is taken into account, but it grows with the time. No detailed studies of the convergence rate for the error term were reported in [18, 19]. 
The purpose of this work is to develop a systematic approach to the derivation and justification of the error terms of the weakly-nonlinear solutions for the regularized Boussinesq equation (1) when the first-order correction is added to the leading-order term. We will also give a systematic comparison of the convergence rates predicted by the theory and observed in numerical simulations.

Our analytical results are obtained in the periodic domain, where derivation and justification of the reduced equations become easy with the use of Fourier series (see [33] for similar derivations). Our numerical examples resemble localized waves on a long but fixed period, which still fits well to the analytical theory. Additional assumptions on the spatial decay of initial data are required to work in the infinite domains. See [3, 4] for recent works on justifications of the uncoupled $\mathrm{KdV}$ equations in the context of the defocussing nonlinear Schrödinger equations in the infinite domain.

The novelties of our paper (compared to the results obtained in [7], [18, 19], and [42]) are the following. First, we discuss in details the role of the nonzero mean value of the periodic solutions in the justification analysis. For $\delta=0$, nonzero mean values were found to degrade the accuracy of the long-wave approximation [7] because no corrections to the wave speeds were introduced in the uncoupled $\mathrm{KdV}$ equations. For $\delta>0$, all solutions of the Ostrovsky equations must satisfy the zero-mean constraint [2] but the zero-mean constraint does not have to be assumed for the regularized Boussinesq equation (1). We will show how the mean value can be incorporated naturally in the long-wave approximation for the regularized Boussinesq equation (1) both for $\delta=0$ and $\delta>0$.

The other novelty of our work is that we extend the analysis to justify the first-order correction terms to the long-wave approximation on the time scale of the Ostrovsky equation. The first-order correction terms were written explicitly in the previous works [18, 19, but the validity of these terms at times greater than order one can only be achieved if these terms satisfy the linearized Ostrovsky equation. The linearized KdV equations have also appeared in [42] in the framework of the regularized Boussinesq equation (with $\delta=0$ ). Compared to this work, we give precise expressions in terms of solutions of the leading order equations to solve the associated initial-value problem up to the first-order correction terms and develop the justification analysis in the energy space of the regularized Boussinesq equation.

Before closing the introduction, we shall also discuss the reductive perturbation schemes, which were used to obtain the integrable $\mathrm{KdV}$ hierarchy from the shallow water-wave and Boussinesq equations [23, 24, 25]. It was later shown in [17, 22] that there are obstacles to the asymptotic integrability of the original physical equations reduced to the integrable $\mathrm{KdV}$ hierarchy in the sense that the formal asymptotic expansions become non-uniform at higher orders of $\epsilon$. Within the framework of our approach, we do not need to set up the time evolution along higher flows of the KdV hierarchy if we are only interested in the validity of the first-order correction terms at the time scale of the $\mathrm{KdV}$ equation. In other words, we can fully control the approximation error within the required order of accuracy of the asymptotic expansions without analyzing the secular terms in the linearized KdV equations and the related difference between asymptotically integrable and non-integrable perturbation terms.

This paper is organized as follows. In Section 2, we set up the long-wave scaling and analyze dynamics of the nonzero mean value of periodic functions. In Section 3, we describe 
the formal asymptotic theory and prove the justification result about the approximation error of the asymptotic expansion, in the framework of the regularized Boussinesq equation with $\delta=0$. Section 4 extends analysis to the case of the regularized Boussinesq equation with $\delta>0$. Section 5 illustrates the main results with numerical computations. Section 6 concludes the paper.

\section{LONG-WAVE SCALING AND DYNAMiCS OF THE MEAN VALUE}

Using the scaling transformation,

$$
u(x, t)=\epsilon U(x, t), \quad x=\sqrt{\epsilon} \xi, \quad t=\sqrt{\epsilon} \tau, \quad \delta=\epsilon^{2} \gamma, \quad \epsilon>0,
$$

we rewrite the regularized Boussinesq equation (1) in the equivalent form:

$$
U_{t t}-U_{x x}=\epsilon\left(\frac{1}{2}\left(U^{2}\right)_{x x}+U_{t t x x}-\gamma U\right),
$$

Throughout this paper, we will interpret the right-hand side as a $\mathcal{O}(\epsilon)$ perturbation and develop the asymptotic theory in the limit of small $\epsilon$. In physical settings, the perturbed wave equation (2) has also the $\mathcal{O}\left(\epsilon^{2}\right)$ correction terms, which need to be incorporated in the asymptotic theory. However, these modifications are technically straightforward and obey the same justification analysis, hence we are going to neglect the $\mathcal{O}\left(\epsilon^{2}\right)$ terms in the Boussinesq equation (2).

We consider the initial-value problem with the initial data

$$
\left.U\right|_{t=0}=F(x),\left.\quad U_{t}\right|_{t=0}=V(x),
$$

where the given functions $F$ and $V$ are in the class of squared integrable $(2 L)$-periodic functions. We can expand them into the Fourier series

$$
F(x)=\sum_{n \in \mathbb{Z}} F_{n} e^{\frac{i \pi n x}{L}}, \quad V(x)=\sum_{n \in \mathbb{Z}} V_{n} e^{\frac{i \pi n x}{L}} .
$$

For the sake of simplification, we assume that $F$ and $V$ are $\epsilon$-independent, although extension to a general case is also straightforward.

The following local existence result is similar to the local well-posedness theory for regularized Boussinesq systems [5, 6].

Proposition 1. Fix $s>\frac{1}{2}$. For any $(F, V) \in H_{\mathrm{per}}^{s}(-L, L) \times H_{\mathrm{per}}^{s}(-L, L)$, there exists an $\epsilon$-independent $t_{0}>0$ and a unique solution $U(t) \in C^{1}\left(\left[0, t_{0}\right], H_{\mathrm{per}}^{s}(-L, L)\right)$ of the regularized Boussinesq equation (2) with any $\epsilon>0$ and $\gamma \geq 0$.

Proof. The evolution problem can be written in the operator form:

$$
U_{t t}-L_{\epsilon} U_{x x}+\epsilon \gamma L_{\epsilon} U=M_{\epsilon} U^{2},
$$

where

$$
L_{\epsilon}:=\left(1-\epsilon \partial_{x}^{2}\right)^{-1}, \quad M_{\epsilon}:=\frac{1}{2} \epsilon \partial_{x}^{2} L_{\epsilon} .
$$

By using Fourier series, we realize that both operators $L_{\epsilon}$ and $M_{\epsilon}$ are bounded for any $\epsilon>0$ and $\gamma \geq 0$ with the $\epsilon$-independent bounds:

$$
\left\|L_{\epsilon} U\right\|_{L_{\mathrm{per}}^{2}} \leq\|U\|_{L_{\mathrm{per}}^{2}}, \quad\left\|M_{\epsilon} U\right\|_{L_{\mathrm{per}}^{2}} \leq \frac{1}{2}\|U\|_{L_{\mathrm{per}}^{2}} .
$$


Using Duhamel's principle, we rewrite the evolution problem (5) with the initial data (3) in the equivalent integral form:

$$
U(t)=S_{t}(t) \star F+S(t) \star V+\int_{0}^{t} S(t-\tau) \star\left(M_{\epsilon} U^{2}(\tau)-\epsilon \gamma L_{\epsilon} U(\tau)\right) d \tau,
$$

where the star denotes the convolution operator and $S(t)$ is the fundamental solution operator with the Fourier image:

$$
\hat{S}(t)=\frac{\sin (k \hat{\ell}(k) t)}{k \hat{\ell}(k)}, \quad \ell(k):=\frac{1}{\sqrt{1+\epsilon k^{2}}} .
$$

Because $S(t)$ and $S_{t}(t)$ are bounded operators from $L_{\text {per }}^{2}(-L, L)$ to $L_{\text {per }}^{2}(-L, L)$ for any $t \in \mathbb{R}$, the fixed-point iteration method (see, e.g., [8]) implies that there exists a unique local solution of the integral equation (5) in the class

$$
U(t) \in C\left(\left[0, t_{0}\right], H_{\mathrm{per}}^{s}(-L, L)\right)
$$

for any $(F, V) \in H_{\text {per }}^{s}(-L, L) \times H_{\text {per }}^{s}(-L, L)$ and any $s>\frac{1}{2}$, where $t_{0}>0$ is an $\epsilon$-independent local existence time. On the other hand, $U_{t}(t)$ is defined by differentiation of the integral equation (5):

$$
U_{t}(t)=S_{t t}(t) \star F+S_{t}(t) \star V+\int_{0}^{t} S_{t}(t-\tau) \star\left(M_{\epsilon} U^{2}(\tau)-\epsilon \gamma L_{\epsilon} U(\tau)\right) d \tau .
$$

Because $S_{t t}(t)$ satisfies the bound

$$
\left\|S_{t t}(t) \star F\right\|_{L_{\mathrm{per}}^{2}} \leq \frac{1}{\sqrt{\epsilon}}\|F\|_{L_{\mathrm{per}}^{2}}, \quad \epsilon>0
$$

and $U(t)$ is defined in the class $(7)$, we have $U_{t}(t) \in C\left(\left[0, t_{0}\right], H_{\mathrm{per}}^{s}(-L, L)\right)$ for any $\epsilon>0$. As a result, $U(t) \in C^{1}\left(\left[0, t_{0}\right], H_{\text {per }}^{s}(-L, L)\right)$.

In the long-wave approximation, we will need to extend local solutions of the Boussinesq equation (2) in the energy space to the time intervals with $t_{0}=\mathcal{O}\left(\epsilon^{-1}\right)$. This continuation is achieved with energy methods resulting in the following wave breaking criterion.

Proposition 2. Let $U(t) \in C^{1}\left(\left[0, t_{0}\right], H_{\mathrm{per}}^{1}(-L, L)\right)$ be a local solution in Proposition 1 . The solution is extended to the time interval $\left[0, t_{0}^{\prime}\right]$ with $t_{0}^{\prime}>t_{0}$ if

$$
M:=\sup _{t \in\left[0, t_{0}^{\prime}\right]}\|U(t)\|_{L_{\text {per }}^{\infty}}+\sup _{t \in\left[0, t_{0}^{\prime}\right]}\left\|U_{t}(t)\right\|_{L_{\text {per }}^{\infty}}<\infty .
$$

Proof. Let us define the energy function

$$
E(U):=\int_{-L}^{L}\left(U_{t}^{2}+U_{x}^{2}+\epsilon \gamma U^{2}+\epsilon U_{t x}^{2}+\epsilon U U_{x}^{2}\right) d x,
$$

for any local solution $U(t) \in C^{1}\left(\left[0, t_{0}\right], H_{\text {per }}^{1}(-L, L)\right)$. Multiplying the Boussinesq equation (2) by $U_{t}$ and integrating by parts, we obtain

$$
\frac{d E(U)}{d t}=\epsilon \int_{-L}^{L} U_{t} U_{x}^{2} d x+\left.2\left(U_{t} U_{x}+\epsilon U_{t} U_{t t x}+\epsilon U U_{t} U_{x}\right)\right|_{x=-L} ^{x=L} .
$$


By standard approximation arguments in Sobolev space $H_{\mathrm{per}}^{2}(-L, L)$, the trace of the boundary values is zero and we obtain a priori energy estimate

$$
\frac{d E(U)}{d t}=\epsilon \int_{-L}^{L} U_{t} U_{x}^{2} d x \leq \epsilon\left\|U_{t}\right\|_{L_{\mathrm{per}}^{\infty}}\left\|U_{x}\right\|_{L_{\mathrm{per}}^{2}}^{2} .
$$

Under the condition (8), there is a positive constant $C(\epsilon M)$ that depends on $\epsilon M$ such that

$$
\left\|U_{x}\right\|_{L_{\mathrm{per}}^{2}}^{2} \leq C(\epsilon M) E(U)
$$

By Gronwall's inequality, we then obtain

$$
E(U) \leq E\left(U_{0}\right) e^{\epsilon M C(\epsilon M) t}, \quad t \in\left[0, t_{0}^{\prime}\right]
$$

such that the solution is extended to the time $t_{0}^{\prime}>t_{0}$ in the energy space, that is, in the class $U(t) \in C^{1}\left(\left[0, t_{0}^{\prime}\right], H_{\mathrm{per}}^{1}(-L, L)\right)$.

Remark 1. By Sobolev embedding of $H_{\mathrm{per}}^{1}(-L, L)$ to $L_{\mathrm{per}}^{\infty}(-L, L)$, we have $M=\mathcal{O}\left(\epsilon^{-1 / 2}\right)$ and $C(\epsilon M)=\mathcal{O}(1)$ as $\epsilon \rightarrow 0$. The energy method of Proposition 2 guarantees continuation of the local solution of Proposition 1 to the time intervals of $t_{0}=\mathcal{O}\left(\epsilon^{-1 / 2}\right)$. However, this is not sufficient as the long-wave approximation requires us to continue the solution to the time intervals of $t_{0}=\mathcal{O}\left(\epsilon^{-1}\right)$. We achieve this goal by controlling $M$ with the $\mathcal{O}(1)$ bound as $\epsilon \rightarrow 0$ (see the proof of Theorem 1 below).

Remark 2. D'Alembert solution of the wave equation $U_{t t}-U_{x x}=0$ (for $\epsilon=0$ ) only requires us to set

$$
(F, V) \in H_{\mathrm{per}}^{1}(-L, L) \times L_{\mathrm{per}}^{2}(-L, L)
$$

to have $U \in C\left(\mathbb{R}, H_{\text {per }}^{1}(-L, L)\right) \cap C^{1}\left(\mathbb{R}, L_{\text {per }}^{2}(-L, L)\right)$. However, we actually need to find a solution in the class $U \in C^{1}\left(\mathbb{R}, H_{\mathrm{per}}^{1}(-L, L)\right)$ in order to bound all terms in the energy norm (9). This is achieved in Proposition 1, which gives an improved local-wellposedness result for the regularized Boussinesq equation (2).

We shall now study the dynamics of the mean value of the $(2 L)$-periodic solution $U(t) \in$ $C^{1}\left(\left[0, t_{0}\right], H_{\mathrm{per}}^{1}(-L, L)\right)$. Integrating equation (2) in $x$ over the period $(2 L)$ in the class of sufficiently smooth $(2 L)$-periodic functions, we obtain the evolution equation for the mean value:

$$
\frac{d^{2}}{d t^{2}} \int_{-L}^{L} U(x, t) d x=-\epsilon \gamma \int_{-L}^{L} U(x, t) d x
$$

which shows that

$$
\langle U\rangle(t):=\frac{1}{2 L} \int_{-L}^{L} U(x, t) d x=F_{0} \cos (\sqrt{\epsilon \gamma} t)+V_{0} \frac{\sin (\sqrt{\epsilon \gamma} t)}{\sqrt{\epsilon \gamma}},
$$

where $F_{0}$ and $V_{0}$ are mean values of the Fourier series (4).

To eliminate the linear growth of the mean value $\langle U\rangle$ in $t$ for $\gamma=0$, we should assume that $V_{0}=0$ (that is, $V$ has zero mean value). If $\gamma>0$, the mean value $\langle U\rangle$ is oscillating with the frequency $\omega=(\epsilon \gamma)^{1 / 2}$, and hence $F_{0}$ and $V_{0}$ can be nonzero in general. However, for any $t \in \mathbb{R}$, the mean value $\langle U\rangle$ diverges as $\mathcal{O}\left(\epsilon^{-1 / 2}\right)$ if $V_{0} \neq 0$. Therefore, in both cases 
$\gamma=0$ and $\gamma>0$, we would like to eliminate the secular growth of the mean value $\langle U\rangle$ in $t$ by requiring that

$$
V_{0}=\frac{1}{2 L} \int_{-L}^{L} V(x) d x=0 .
$$

In this case, the mean value $\langle U\rangle$ is bounded in $t \in \mathbb{R}$ and $\epsilon \in \mathbb{R}_{+}$with a uniform limit as $\epsilon \rightarrow 0$ for any $\gamma \geq 0$. For many physical applications, the constraint $V_{0}=0$ is rather natural in the physical contexts of the regularized Boussinesq equation (2) both for $\gamma=0$ and $\gamma>0$.

\section{LONG-WAVE APPROXIMATION FOR $\gamma=0$}

We shall consider the initial-value problem for the regularized Boussinesq equation,

$$
U_{t t}-U_{x x}=\epsilon\left(\frac{1}{2}\left(U^{2}\right)_{x x}+U_{t t x x}\right),
$$

The initial data are given by (3) and (4) subject to the zero-mean velocity constraint (12). By the exact solution (11), the mean value of the solution $U$ is constant in $t$ with $\langle U\rangle=F_{0}$.

Substituting $U(x, t)=c_{0}+\tilde{U}(x, t)$ into the regularized Boussinesq equation (13), where $c_{0}:=F_{0}$ and $\tilde{U}$ is the zero-mean part of the $2 L$-periodic function $U$, we obtain the evolution equation

$$
\tilde{U}_{t t}-\tilde{U}_{x x}=\epsilon\left(c_{0} \tilde{U}_{x x}+\frac{1}{2}\left(\tilde{U}^{2}\right)_{x x}+\tilde{U}_{t t x x}\right)
$$

By Proposition 1, there exists a unique local solution $\tilde{U} \in C^{1}\left(\left[0, t_{0}\right], H_{\text {per }}^{s}(-L, L)\right)$ of the evolution equation 14 for any $(\tilde{F}, \tilde{V}) \in H_{\text {per }}^{s}(-L, L) \times H_{\text {per }}^{s}(-L, L)$ with $s>\frac{1}{2}$, where $t_{0}>0$ is a local existence time and the tilded variables denote the zero-mean part of the $(2 L)$-periodic functions.

3.1. Derivation. We shall look for a formal asymptotic solution of the evolution equation (14) up to and including the $\mathcal{O}\left(\epsilon^{2}\right)$ terms:

$$
\tilde{U}(x, t)=U_{0}(x, t)+\epsilon U_{1}(x, t)+\epsilon^{2} U_{2}(x, t)+\mathcal{O}\left(\epsilon^{3}\right) .
$$

In the formal theory, we collect together terms at each order.

Order $\mathcal{O}\left(\epsilon^{0}\right)$ : The leading order $U_{0}$ satisfies the initial-value problem for the wave equation:

$$
\left\{\begin{array}{l}
\left(\partial_{t}^{2}-\partial_{x}^{2}\right) U_{0}=0 \\
\left.U_{0}\right|_{t=0}=\tilde{F} \\
\left.\partial_{t} U_{0}\right|_{t=0}=\tilde{V}
\end{array}\right.
$$

The d'Alembert solution of the wave equation (16) consists of a superposition of two counterpropagating waves of zero mean:

$$
U_{0}(x, t)=f^{-}\left(\xi_{-}\right)+f^{+}\left(\xi_{+}\right), \quad \xi_{ \pm}=x \pm t,
$$


where

$$
f^{ \pm}\left(\xi_{ \pm}\right)=\frac{1}{2} \tilde{F}\left(\xi_{ \pm}\right) \pm \frac{1}{2} \partial_{\xi_{ \pm}}^{-1} \tilde{V}\left(\xi_{ \pm}\right)=\frac{1}{2} \sum_{n \in \mathbb{Z} \backslash\{0\}}\left(F_{n} \pm \frac{L V_{n}}{\pi i n}\right) e^{\frac{i \pi n \xi_{ \pm}}{L}}
$$

and $\partial_{\xi_{ \pm}}^{-1}$ denote the zero-mean anti-derivative of the zero-mean periodic functions.

Order $\mathcal{O}(\epsilon)$ : At this point, we should realize that the next-order correction terms are going to grow linearly in time $t$ unless we will modify the leading-order solution on a slow time scale $T=\epsilon t$. Therefore, we modify the leading-order solution (17) with the slow time variable:

$$
U_{0}(x, t)=f^{-}\left(\xi_{-}, T\right)+f^{+}\left(\xi_{+}, T\right), \quad f^{ \pm}\left(\xi_{ \pm}, T\right)=\sum_{n \in \mathbb{Z} \backslash\{0\}} a_{n}^{ \pm}(T) e^{\frac{i \pi n \xi_{ \pm}}{L}}
$$

where

$$
\left.a_{n}^{ \pm}\right|_{T=0}=\frac{1}{2}\left(F_{n} \pm \frac{L V_{n}}{\pi i n}\right), \quad n \in \mathbb{Z} \backslash\{0\} .
$$

We know the initial data for $f^{ \pm}$in slow time $T=\epsilon t$, but we do not know yet the evolution equations for $f^{ \pm}\left(\xi_{ \pm}, T\right)$. To derive these equations, we consider the first-order correction terms:

$$
\left\{\begin{array}{l}
\left(\partial_{t}^{2}-\partial_{x}^{2}\right) U_{1}=-2 \partial_{t T}^{2} U_{0}+c_{0} \partial_{x}^{2} U_{0}+\frac{1}{2} \partial_{x}^{2}\left(U_{0}^{2}\right)+\partial_{t t x x}^{4} U_{0} \\
\left.U_{1}\right|_{t=0}=0 \\
\left.\partial_{t} U_{1}\right|_{t=0}=-\left.\partial_{T} U_{0}\right|_{t=0}
\end{array}\right.
$$

Using the Fourier series, $U_{1}(x, t)=\sum_{n \in \mathbb{Z} \backslash\{0\}} g_{n}(t) e^{\frac{i \pi n x}{L}}$, we reduce the evolution equation in the system (21) to the uncoupled system of differential equations:

$$
\frac{d^{2} g_{n}}{d t^{2}}+\left(\frac{\pi n}{L}\right)^{2} g_{n}=h_{n}(t)
$$

where

$$
\begin{aligned}
h_{n}(t)= & -\frac{2 \pi i n}{L}\left(\frac{d a_{n}^{+}}{d T} e^{\frac{i \pi n t}{L}}-\frac{d a_{n}^{-}}{d T} e^{-\frac{i \pi n t}{L}}\right)+\frac{\pi^{4} n^{4}}{L^{4}}\left(a_{n}^{+} e^{\frac{i \pi n t}{L}}+a_{n}^{-} e^{-\frac{i \pi n t}{L}}\right) \\
& -\frac{\pi^{2} n^{2}}{L^{2}} c_{0}\left(a_{n}^{+} e^{\frac{i \pi n t}{L}}+a_{n}^{-} e^{-\frac{i \pi n t}{L}}\right)-\frac{\pi^{2} n^{2}}{L^{2}}\left(\sum_{k \in \mathbb{Z} \backslash\{0, n\}} a_{k}^{+} a_{n-k}^{-} e^{\frac{i \pi(2 k-n) t}{L}}\right) \\
& -\frac{\pi^{2} n^{2}}{2 L^{2}}\left(\sum_{k \in \mathbb{Z} \backslash\{0, n\}} a_{k}^{+} a_{n-k}^{+}\right) e^{\frac{i \pi n t}{L}}-\frac{\pi^{2} n^{2}}{2 L^{2}}\left(\sum_{k \in \mathbb{Z} \backslash\{0, n\}} a_{k}^{-} a_{n-k}^{-}\right) e^{-\frac{i \pi n t}{L}} .
\end{aligned}
$$

The terms $e^{ \pm \frac{i \pi n t}{L}}$ in the right-hand-side of differential equations 22 are resonant (that is, they induce a linear growth of $g_{n}(t)$ in a fast time $\left.t\right)$. To remove these resonant terms, we define uniquely the evolution problem for the Fourier coefficients of the leading-order 
solution (19):

$$
\mp \frac{2 \pi i n}{L} \frac{d a_{n}^{ \pm}}{d T}+\frac{\pi^{4} n^{4}}{L^{4}} a_{n}^{ \pm}-\frac{\pi^{2} n^{2}}{L^{2}} c_{0} a_{n}^{ \pm}-\frac{\pi^{2} n^{2}}{2 L^{2}} \sum_{k \in \mathbb{Z} \backslash\{0, n\}} a_{k}^{ \pm} a_{n-k}^{ \pm}=0,
$$

subject to the initial conditions 20 .

In the equivalent differential form, the evolution problem (23) coincides with the two uncoupled KdV equations

$$
\frac{\partial}{\partial \xi_{ \pm}}\left(\mp 2 \frac{\partial f^{ \pm}}{\partial T}+\frac{\partial^{3} f^{ \pm}}{\partial \xi_{ \pm}^{3}}+c_{0} \frac{\partial f^{ \pm}}{\partial \xi_{ \pm}}+f^{ \pm} \frac{\partial f^{ \pm}}{\partial \xi_{ \pm}}\right)=0 .
$$

We consider the initial-value problem for the uncoupled KdV equations (24) starting with the initial values $\left.f^{ \pm}\right|_{T=0}$ given by $(18)$. By the local and global well-posedness theory for the $\mathrm{KdV}$ equation [9], there exist unique global solutions $f^{ \pm} \in C\left(\mathbb{R}_{+}, H_{\text {per }}^{s}(-L, L)\right)$ of the $\mathrm{KdV}$ equations 24 for any $\left.f^{ \pm}\right|_{T=0} \in H_{\text {per }}^{s}(-L, L)$ with $s \geq-\frac{1}{2}$.

After the constraints (23) are substituted back into the differential equations (22), we obtain the linear inhomogeneous equations

$$
\frac{d^{2} g_{n}}{d t^{2}}+\left(\frac{\pi n}{L}\right)^{2} g_{n}=-\frac{\pi^{2} n^{2}}{L^{2}} \sum_{k \in \mathbb{Z} \backslash\{0, n\}} a_{k}^{+} a_{n-k}^{-} e^{\frac{i \pi(2 k-n) t}{L}},
$$

subject to the initial conditions

$$
g_{n}(0)=0, \quad \partial_{t} g_{n}(0)=-\partial_{T} a_{n}^{+}(0)-\partial_{T} a_{n}^{-}(0) .
$$

This initial-value problem admits the following bounded solution:

$$
g_{n}(t)=\sum_{k \in \mathbb{Z} \backslash\{0, n\}} \frac{n^{2}}{4 k(k-n)} a_{k}^{+} a_{n-k}^{-} e^{\frac{i \pi(2 k-n) t}{L}}+G_{n} \cos \left(\frac{\pi n t}{L}\right)+H_{n} \sin \left(\frac{\pi n t}{L}\right),
$$

where $G_{n}$ and $H_{n}$ are constants of integrations to be found from the initial conditions for $g_{n}$. Using the previous expression for $g_{n}$, we rewrite the first-order correction term in the implicit form:

$$
U_{1}(x, t)=f_{c}(x, t)+\phi^{-}\left(\xi_{-}, T\right)+\phi^{+}\left(\xi_{+}, T\right),
$$

where $f_{c}$ is uniquely defined by

$$
f_{c}(x, t)=\sum_{n \in \mathbb{Z} \backslash\{0\}} \sum_{k \in \mathbb{Z} \backslash\{0, n\}} \frac{n^{2}}{4 k(k-n)} a_{k}^{+}(T) a_{n-k}^{-}(T) e^{\frac{i \pi(2 k-n) t}{L}+\frac{i \pi n x}{L}}
$$

and $\phi^{ \pm}$are counter-propagating waves of the wave equation given by

$$
\phi^{ \pm}\left(\xi_{ \pm}, T\right)=\sum_{n \in \mathbb{Z} \backslash\{0\}} b_{n}^{ \pm}(T) e^{\frac{i \pi n \xi_{ \pm}}{L}} .
$$

An explicit expression for the first-order correction term was derived recently in [18, 19, by averaging the differential equations with respect to the fast time in characteristic coordinates. We check that our expression (26) coincides with the one derived in [18, 19]:

$$
f_{c}(x, t)=-\frac{1}{4}\left(2 f^{+} f^{-}+\left(\partial_{\xi^{+}} f^{+}\right)\left(\partial_{\xi_{-}}^{-1} f^{-}\right)+\left(\partial_{\xi^{-}} f^{-}\right)\left(\partial_{\xi_{+}}^{-1} f^{+}\right)\right)
$$


where $\partial_{\xi_{ \pm}}^{-1} f^{ \pm}$denote again the zero-mean anti-derivatives of the zero-mean periodic functions $f^{ \pm}$.

Using the initial conditions for $g_{n}$, we can express the initial data for the amplitudes $b_{n}^{ \pm}$ explicitly:

$$
\left.b_{n}^{ \pm}\right|_{T=0}=-\left.\left.\sum_{k \in \mathbb{Z} \backslash\{0, n\}} \frac{n(n \pm(2 k-n))}{8 k(k-n)}\left(a_{k}^{+} a_{n-k}^{-}\right)\right|_{T=0} \mp \frac{L}{2 i \pi n}\left(\frac{d a_{n}^{+}}{d T}+\frac{d a_{n}^{-}}{d T}\right)\right|_{T=0}
$$

Order $\mathcal{O}\left(\epsilon^{2}\right)$ : The time evolution of $\phi^{ \pm}$with respect to the slow time $T$ is not defined at the $\mathcal{O}(\epsilon)$ order. To derive the time evolution, we consider the second-order correction terms:

$$
\left\{\begin{array}{l}
\left(\partial_{t}^{2}-\partial_{x}^{2}\right) U_{2}=-2 \partial_{t T}^{2} U_{1}-\partial_{T}^{2} U_{0}+c_{0} \partial_{x}^{2} U_{1}+\partial_{x}^{2}\left(U_{0} U_{1}\right)+\partial_{t t x x}^{4} U_{1}+2 \partial_{t T x x}^{4} U_{0} \\
\left.U_{2}\right|_{t=0}=0 \\
\left.\partial_{t} U_{2}\right|_{t=0}=-\left.\partial_{T} U_{1}\right|_{t=0}
\end{array}\right.
$$

Repeating the procedure of removing the resonant terms $e^{ \pm \frac{i \pi n t}{L}}$, we define uniquely the evolution problem for the Fourier coefficients of the first-order solution (25):

$$
\begin{array}{r}
-\frac{d^{2} a_{n}^{ \pm}}{d T^{2}} \mp \frac{2 \pi i n}{L} \frac{d b_{n}^{ \pm}}{d T}+\frac{\pi^{4} n^{4}}{L^{4}} b_{n}^{ \pm} \mp \frac{2 i \pi^{3} n^{3}}{L^{3}} \frac{d a_{n}^{ \pm}}{d T}-\frac{\pi^{2} n^{2}}{L^{2}} c_{0} b_{n}^{ \pm} \\
-\frac{\pi^{2} n^{2}}{L^{2}} \sum_{k \in \mathbb{Z} \backslash\{0, n\}} a_{k}^{ \pm} b_{n-k}^{ \pm}-\frac{\pi^{2} n^{2}}{L^{2}} \sum_{k \in \mathbb{Z} \backslash\{0, n\}} \frac{(n-k)^{2}}{4 n k} a_{n}^{ \pm}\left|a_{k}^{\mp}\right|^{2}=0,
\end{array}
$$

subject to the initial conditions (29).

In the equivalent differential form, the evolution equations (31) coincide with the linearized $\mathrm{KdV}$ equations

$$
\frac{\partial}{\partial \xi_{ \pm}}\left(\mp 2 \frac{\partial \phi^{ \pm}}{\partial T}+\frac{\partial^{3} \phi^{ \pm}}{\partial \xi_{ \pm}^{3}}+c_{0} \frac{\partial \phi^{ \pm}}{\partial \xi_{ \pm}}+\frac{\partial}{\partial \xi_{ \pm}} f^{ \pm} \phi^{ \pm}\right)=\frac{\partial^{2} f^{ \pm}}{\partial T^{2}} \mp 2 \frac{\partial^{4} f^{ \pm}}{\partial \xi_{ \pm}^{3} T}+\frac{\partial^{2} f_{s}^{ \pm}}{\partial \xi_{ \pm}^{2}}
$$

where

$$
\begin{aligned}
f_{s}^{ \pm}\left(\xi_{ \pm}, T\right) & =-\sum_{n \in \mathbb{Z} \backslash\{0\}} \sum_{k \in \mathbb{Z} \backslash\{0, n\}} \frac{(n-k)^{2}}{4 n k}\left|a_{k}^{\mp}(T)\right|^{2} a_{n}^{ \pm}(T) e^{\frac{i \pi n \xi_{ \pm}}{L}} \\
& =\frac{1}{2} f^{ \pm}\left(\xi_{ \pm}, T\right)\left(\sum_{k \in \mathbb{Z} \backslash\{0\}}\left|a_{k}^{\mp}(T)\right|^{2}\right) \\
& =\frac{1}{4 L} f^{ \pm}\left(\xi_{ \pm}, T\right) \int_{-L}^{L}\left|f^{\mp}(\xi, T)\right|^{2} d \xi .
\end{aligned}
$$

The initial-value problem for the linearized KdV equations (32) starts with the initial values $\left.\phi^{ \pm}\right|_{T=0}$ given by (27) and (29) (the closed form expressions in terms of the leading order solutions $f^{ \pm}$can be found in [18, 19]). There exists a unique global solution $\phi^{ \pm} \in C\left(\mathbb{R}_{+}, H_{\text {per }}^{s}(-L, L)\right)$ for any $\left.\phi^{ \pm}\right|_{T=0} \in H_{\text {per }}^{s}(-L, L)$ with $s \geq-\frac{1}{2}$ provided that the source term on the right-hand-side of (32) is sufficiently smooth in $T$ and $\xi_{ \pm}$. 
After the constraints (31) are substituted back into the differential equations, we can obtain a bounded solution for $U_{2}(x, t)$. This completes the construction of the formal asymptotic expansion 15 up to and including the $\mathcal{O}\left(\epsilon^{2}\right)$ terms.

3.2. Justification. We shall now justify the long-wave approximation. The following theorem gives the main result of the justification analysis.

Theorem 1. Assume that $(F, V) \in H_{\mathrm{per}}^{1}(-L, L) \times H_{\mathrm{per}}^{1}(-L, L)$ subject to the zero-mean constraint (12) on $V$. Fix $s \geq 10$ and let $f^{ \pm} \in C\left(\mathbb{R}, H_{\mathrm{per}}^{s}(-L, L)\right)$ be global solutions of the KdV equations (24) starting with the initial data (18). Let $U_{0}$ and $U_{1}$ be given by (19) and (25) with (26), (27), and (29). There is $\epsilon_{0}>0$ such that for all $\epsilon \in\left(0, \epsilon_{0}\right)$ and all $\epsilon$ independent $T_{0}>0$, there is an $\epsilon$-independent constant $C>0$ such that for all $t_{0} \in\left[0, T_{0} / \epsilon\right]$, the local solution of the regularized Boussinesq equation (13) satisfies

$$
\sup _{t \in\left[0, t_{0}\right]}\left\|U-c_{0}-U_{0}-\epsilon U_{1}\right\|_{H_{\mathrm{per}}^{1}} \leq C \epsilon^{2} t_{0} .
$$

If, in addition, $\phi^{ \pm}$in (27) satisfies the linearized $K d V$ equations (32) subject to the initial data (29) and $s$ is sufficiently large, then for all $\epsilon \in\left(0, \epsilon_{0}\right)$ and all $\epsilon$-independent $T_{0}>0$, there is an $\epsilon$-independent constant $C>0$ such that

$$
\sup _{t \in\left[0, T_{0} / \epsilon\right]}\left\|U-c_{0}-U_{0}-\epsilon U_{1}\right\|_{H_{\mathrm{per}}^{1}} \leq C \epsilon^{2} .
$$

Before proving the theorem, we make some relevant remarks.

Remark 3. The bound (34) of Theorem 1 generalizes the result of Theorem 7 in [7] obtained in the context of Boussinesq systems. However, if the authors of [7] restrict their consideration to the zero mean value for the initial data (case (ii') in Theorem 7) and show that the nonzero mean values do not produce good long-wave approximations (cases (ii) and (iii) in Theorem 7), we show that the mean value in the initial data for $\left.U\right|_{t=0}=F$ can be naturally incorporated in the justification analysis by modifying the velocity term of the uncoupled $K d V$ equations (24).

Remark 4. The difference between bounds (34) and (35) of Theorem 1 is in the time scales, for which the first-order correction terms remain valid. Bound (34) shows that the error of the long-wave approximation is of the $\mathcal{O}\left(\epsilon^{2}\right)$ order at the time scale $t_{0}=\mathcal{O}(1)$ but becomes comparable with the $\mathcal{O}(\epsilon)$ first-order correction terms at the time scale $t_{0}=\mathcal{O}\left(\epsilon^{-1}\right)$. On the other hand, bound (35) shows that the error of the long-wave approximation remains of the $\mathcal{O}\left(\epsilon^{2}\right)$ order at the time scale $t_{0}=\mathcal{O}\left(\epsilon^{-1}\right)$ if the first-order correction terms satisfy the linearized KdV equations (32). This improved result corresponds to Theorem 1.1 in [42] on the infinite line with the only difference that the justification analysis is performed in the energy space of the regularized Boussinesq equation (13) compared to the space $H^{\sigma} \cap H^{\sigma+8}$ with $\sigma \geq 4$ used in [42].

Remark 5. Figures 3(c) and 5(c) in [19] illustrate the growth of the approximation error without the account of the linearized $K d V$ equation (32) at the time scale $t_{0}=\mathcal{O}\left(\epsilon^{-1}\right)$. Despite the fact that the first-order correction terms were found to give a smaller approximation error, 
the comparable $\mathcal{O}(\epsilon)$ behavior between the first-order correction terms and the approximation errors was observed on these figures at the time scale $t_{0}=\mathcal{O}\left(\epsilon^{-1}\right)$.

Remark 6. It is customary to combine (24) and (32) to yields a higher-order KdV equation but the precise form of this higher-order KdV equation is not unique [23, 24, 25]. The problem of non-uniqueness does not occur in the method we are using in our analytical work, where the system of reduced amplitude equations (24) and (32) is uniquely determined for the Cauchy problem associated with the regularized Boussinesq equation (13).

Proof of Theorem 1. We shall first prove bound (34) on the approximation error. We work in energy space of the regularized Boussinesq equation (13) and write the approximation in the form

$$
U(x, t)=c_{0}+U_{0}(x, t)+\epsilon U_{1}(x, t)+\epsilon^{2} \hat{U}(x, t),
$$

where $c_{0}=F_{0}$ is the mean value, $U_{0}$ and $U_{1}$ are given explicitly by (19) and (25) with (26), (27), and (29), $f^{ \pm}$satisfy the uncoupled KdV equations (24), and $U$ is the error term that depends on $\epsilon$. Substituting the decomposition (36) into the regularized Boussinesq equation (13), we obtain the time evolution problem for the error term:

$$
\hat{U}_{t t}-\left(1+\epsilon c_{0}\right) \hat{U}_{x x}-\epsilon \hat{U}_{t t x x}=\epsilon \partial_{x}^{2}\left(U_{0} \hat{U}+\epsilon U_{1} \hat{U}+\frac{1}{2} \epsilon^{2} \hat{U}^{2}\right)+\hat{H},
$$

where the initial data are

$$
\left.\hat{U}\right|_{t=0}=0,\left.\quad \hat{U}_{t}\right|_{t=0}=-\left.\partial_{T} U_{1}\right|_{T=0},
$$

and the source term is

$$
\begin{aligned}
\hat{H}= & -2 \partial_{t} \partial_{T} U_{1}-\partial_{T}^{2} U_{0}-\epsilon \partial_{T}^{2} U_{1}+c_{0} \partial_{x}^{2} U_{1}+\left(\partial_{t}+\epsilon \partial_{T}\right)^{2} \partial_{x}^{2} U_{1}+2 \partial_{t} \partial_{T} \partial_{x}^{2} U_{0}+\epsilon \partial_{T}^{2} \partial_{x}^{2} U_{0} \\
& +\partial_{x}^{2}\left(U_{0} U_{1}\right)+\frac{1}{2} \epsilon \partial_{x}^{2}\left(U_{1}^{2}\right) .
\end{aligned}
$$

We use a priori energy estimates (see, e.g., [31, 32, for similar applications of this technique). By an extension of Proposition 1, there exists a unique solution

$$
\hat{U} \in C^{1}\left(\left[0, t_{0}\right], H_{\mathrm{per}}^{1}(-L, L)\right)
$$

of the perturbed regularized Boussinesq equation (37) for some $\epsilon$-independent $t_{0}>0$ starting with the initial data (38) provided that the source term satisfies

$$
\hat{H} \in C\left(\left[0, t_{0}\right], H_{\mathrm{per}}^{1}(-L, L)\right) .
$$

By looking at the explicit expression for $\hat{H}$, where $U_{0}$ and $U_{1}$ are given by (19) and 25) and $f^{ \pm} \in C\left(\mathbb{R}, H_{\text {per }}^{s}(-L, L)\right)$ are global solutions of the uncoupled KdV equations (24), we realize that the term of the highest regularity is $\partial_{T}^{2} \partial_{x}^{2} U_{1} \sim \partial_{\xi_{ \pm}}^{9} f^{ \pm}$, hence $\hat{H}$ is from the class (39) if $s \geq 10$.

Let us introduce the energy at the local solution $\hat{U} \in C^{1}\left(\left[0, t_{0}\right], H_{\text {per }}^{1}(-L, L)\right)$ of the perturbed regularized Boussinesq equation (37):

$$
\hat{E}=\int_{-L}^{L}\left(\hat{U}_{t}^{2}+\left(1+\epsilon c_{0}\right) \hat{U}_{x}^{2}+\epsilon \hat{U}_{t x}^{2}+\epsilon U_{0} \hat{U}_{x}^{2}+2 \epsilon U_{0 x} \hat{U} \hat{U}_{x}\right) d x .
$$


Multiplying (37) by $\hat{U}_{t}$, integrating in $x$ over $[-L, L]$, and using approximation arguments in Sobolev spaces of higher regularity, we obtain

$$
\frac{1}{2} \frac{d \hat{E}}{d t}=\int_{-L}^{L}\left[\hat{H} \hat{U}_{t}+\epsilon\left(U_{0 x t} \hat{U} \hat{U}_{x}+U_{0 x} \hat{U}_{t} \hat{U}_{x}+\frac{1}{2} U_{0 t} \hat{U}_{x}^{2}\right)-\epsilon^{2} \hat{U}_{t x}\left(U_{1} \hat{U}+\frac{1}{2} \epsilon \hat{U}^{2}\right)\right] d x .
$$

Because $x$ and $t$ derivatives of $U_{0}$ are $\epsilon$-independent, for sufficiently small $\epsilon$, there is an $\epsilon$-independent positive constant $C$ such that

$$
\left\|\hat{U}_{t}\right\|_{L_{\mathrm{per}}^{2}}^{2}+\left\|\hat{U}_{x}\right\|_{L_{\mathrm{per}}^{2}}^{2}+\epsilon\left\|\hat{U}_{x t}\right\|_{L_{\mathrm{per}}^{2}}^{2} \leq C \hat{E} .
$$

By Poincaré's inequality for $(2 L)$-periodic mean-zero functions, there is another positive constant $C$ such that

$$
\|\hat{U}\|_{L_{\text {per }}^{2}}^{2} \leq C\left\|\hat{U}_{x}\right\|_{L_{\text {per }}^{2}}^{2} \leq C \hat{E} .
$$

By Cauchy-Schwarz's inequality, we obtain from the energy balance equation that

$$
\frac{1}{2} \frac{d \hat{E}}{d t} \leq\|\hat{H}\|_{L_{\mathrm{per}}^{2}}\left\|\hat{U}_{t}\right\|_{L_{\mathrm{per}}^{2}}+C \epsilon\left(\left\|U_{0}\right\|_{L_{\mathrm{per}}^{\infty}}+\epsilon^{1 / 2}\left\|U_{1}\right\|_{L_{\mathrm{per}}^{\infty}}+\epsilon^{3 / 2}\|\hat{U}\|_{L_{\mathrm{per}}^{\infty}}\right) \hat{E},
$$

where the positive constant $C$ is $\epsilon$-independent. Note that the terms $\left\|U_{0 t}\right\|_{L_{\text {per }}^{\infty}},\left\|U_{0 x}\right\|_{L_{\text {per }}^{\infty}}$, $\left\|U_{0 x t}\right\|_{L_{\mathrm{per}}^{\infty}}$, and $\left\|U_{1 x}\right\|_{L_{\mathrm{per}}^{\infty}}$ are not listed in the inequality (41) because they are $\epsilon$-independent and bounded if $f^{ \pm} \in C\left(\mathbb{R}, H_{\text {per }}^{s}(-L, L)\right)$ with $s \geq 10$.

Setting $\hat{E}:=\hat{Q}^{2}$ and using Sobolev's embedding $\|\hat{U}\|_{L_{\text {per }}^{\infty}} \leq C_{\text {emb }} \hat{Q}$, we rewrite a priori energy estimate 41 in the form

$$
\frac{d \hat{Q}}{d t} \leq\|\hat{H}\|_{L_{\mathrm{per}}^{2}}+C \epsilon\left(\left\|U_{0}\right\|_{L_{\mathrm{per}}^{\infty}}+\epsilon^{1 / 2}\left\|U_{1}\right\|_{L_{\mathrm{per}}^{\infty}}+\epsilon^{3 / 2} \hat{Q}\right) \hat{Q}
$$

for another positive $\epsilon$-independent constant $C$. By Gronwall's inequality, we integrate the a priori energy estimate (42) to obtain

$$
\hat{Q}(t) \leq\left(\hat{Q}(0)+t_{0} \sup _{t \in\left[0, t_{0}\right]}\|\hat{H}\|_{L_{\mathrm{per}}^{2}}\right) e^{C \epsilon t}, \quad t \in\left[0, t_{0}\right]
$$

for any $t_{0}>0$, sufficiently small $\epsilon$, and some $\left(t_{0}, \epsilon\right)$-independent positive constant $C_{0}$. Since $\hat{Q}(0)=\left\|\partial_{T} U_{1}\right\|_{L_{\mathrm{per}}^{2}}$, bound 443 yields the result (34) after returning to the original variables (36). By Proposition 2, the solution is continued from $t_{0}=\mathcal{O}(1)$ to $t_{0}=\mathcal{O}\left(\epsilon^{-1}\right)$ thanks to $\hat{Q}(t)<\infty$ for all $t \in\left[0, t_{0}\right]$ and all sufficiently small $\epsilon>0$, as well as to Sobolev's embedding

$$
\left\|\epsilon^{2} \hat{U}\right\|_{L_{\mathrm{per}}^{\infty}} \leq C_{\mathrm{emb}} \epsilon^{2} \hat{Q}, \quad\left\|\epsilon^{2} \hat{U}_{t}\right\|_{L_{\mathrm{per}}^{\infty}} \leq C_{\mathrm{emb}} \epsilon^{3 / 2} \hat{Q} .
$$

Bound $(35)$ is proved similarly after writing $\hat{U}=U_{2}+\epsilon \check{U}$, where $U_{2}$ is a bounded solution of system (30), whereas $\phi^{ \pm}$in (27) satisfy the linearized KdV equations (32) subject to the initial data (29). The new error term $\check{U}$ satisfies a priori energy estimate similar to 42 with the new error term. This energy estimate yields bound (35) after returning to the original variables (36) thanks to the triangle inequality and the bound $\left\|U_{2}\right\|_{H_{\mathrm{per}}^{1}} \leq C$, where the positive constant $C$ is independent of $t_{0}=\mathcal{O}\left(\epsilon^{-1}\right)$ and $\epsilon$. 


\section{LONG-WAVE APPROXIMATION FOR $\gamma>0$}

We shall consider here the case of the regularized Boussinesq equation (2) with $\gamma>0$. We consider again the initial-value problem starting with the initial data (3) and (4) satisfying the zero-mean velocity constraint (12). By the exact solution (11), the mean value of the solution is oscillating in $t$ with $\langle U\rangle=F_{0} \cos (\sqrt{\epsilon \gamma} t)$.

Substituting $U(x, t)=c_{0} \cos (\omega t)+\tilde{U}(x, t)$ into the evolution equation (2), where $c_{0}:=F_{0}$, $\omega:=\sqrt{\epsilon \gamma}$, and $\tilde{U}$ is the zero-mean part of the $2 L$-periodic function $U$, we obtain the evolution equation

$$
\tilde{U}_{t t}-\tilde{U}_{x x}=\epsilon\left(c_{0} \cos (\omega t) \tilde{U}_{x x}+\frac{1}{2}\left(\tilde{U}^{2}\right)_{x x}+\tilde{U}_{t t x x}-\gamma \tilde{U}\right)
$$

By Proposition 1, there exists a unique local solution $\tilde{U} \in C^{1}\left(\left[0, t_{0}\right], H_{\text {per }}^{s}(-L, L)\right)$ of the evolution equation (44) for any $(\tilde{F}, \tilde{V}) \in H_{\text {per }}^{s}(-L, L) \times H_{\text {per }}^{s}(-L, L)$ with $s>\frac{1}{2}$, where $t_{0}>0$ is a local existence time.

4.1. Derivation. We shall repeat steps of the formal asymptotic theory, which relies on the decomposition (15) and the leading-order approximation (19), with the initial conditions (20). In what follows, we first work implicitly with $\epsilon$-dependent $\omega$ and then estimate the size of the correction terms by using the explicit dependence $\omega=\sqrt{\epsilon \gamma}$.

Order $\mathcal{O}(\epsilon)$ : The first-order correction term satisfies the initial-value problem:

$$
\left\{\begin{array}{l}
\left(\partial_{t}^{2}-\partial_{x}^{2}\right) U_{1}=-2 \partial_{t T}^{2} U_{0}+c_{0} \cos (\omega t) \partial_{x}^{2} U_{0}+\frac{1}{2} \partial_{x}^{2}\left(U_{0}^{2}\right)+\partial_{t t x x}^{4} U_{0}-\gamma U_{0} \\
\left.U_{1}\right|_{t=0}=0 \\
\left.\partial_{t} U_{1}\right|_{t=0}=-\left.\partial_{T} U_{0}\right|_{t=0}
\end{array}\right.
$$

Using the Fourier series $U_{1}(x, t)=\sum_{n \in \mathbb{Z} \backslash\{0\}} g_{n}(t) e^{\frac{i \pi n x}{L}}$, we obtain the uncoupled system of differential equations

$$
\frac{d^{2} g_{n}}{d t^{2}}+\left(\frac{\pi n}{L}\right)^{2} g_{n}=h_{n}(t)
$$

where

$$
\begin{aligned}
h_{n}(t)= & -\frac{2 \pi i n}{L}\left(\frac{d a_{n}^{+}}{d T} e^{\frac{i \pi n t}{L}}-\frac{d a_{n}^{-}}{d T} e^{-\frac{i \pi n t}{L}}\right)+\frac{\pi^{4} n^{4}}{L^{4}}\left(a_{n}^{+} e^{\frac{i \pi n t}{L}}+a_{n}^{-} e^{-\frac{i \pi n t}{L}}\right) \\
& -\frac{\pi^{2} n^{2}}{L^{2}} c_{0} \cos (\omega t)\left(a_{n}^{+} e^{\frac{i \pi n t}{L}}+a_{n}^{-} e^{-\frac{i \pi n t}{L}}\right)-\gamma\left(a_{n}^{+} e^{\frac{i \pi n t}{L}}+a_{n}^{-} e^{-\frac{i \pi n t}{L}}\right) \\
& -\frac{\pi^{2} n^{2}}{2 L^{2}}\left(\sum_{k \in \mathbb{Z} \backslash\{0, n\}} a_{k}^{+} a_{n-k}^{+}\right) e^{\frac{i \pi n t}{L}}-\frac{\pi^{2} n^{2}}{2 L^{2}}\left(\sum_{k \in \mathbb{Z} \backslash\{0, n\}} a_{k}^{-} a_{n-k}^{-}\right) e^{-\frac{i \pi n t}{L}} \\
& -\frac{\pi^{2} n^{2}}{L^{2}}\left(\sum_{k \in \mathbb{Z} \backslash\{0, n\}} a_{k}^{+} a_{n-k}^{-} e^{\frac{i \pi(2 k-n) t}{L}}\right) .
\end{aligned}
$$


The resonant terms at $e^{ \pm \frac{i \pi n t}{L}}$ are removed if the Fourier coefficients satisfy the evolution equations:

$$
\mp \frac{2 \pi i n}{L} \frac{d a_{n}^{ \pm}}{d T}+\frac{\pi^{4} n^{4}}{L^{4}} a_{n}^{ \pm}-\gamma a_{n}^{ \pm}-\frac{\pi^{2} n^{2}}{2 L^{2}} \sum_{k \in \mathbb{Z} \backslash\{0, n\}} a_{k}^{ \pm} a_{n-k}^{ \pm}=0,
$$

which are equivalent to the two uncoupled Ostrovsky equations

$$
\frac{\partial}{\partial \xi_{ \pm}}\left(\mp 2 \frac{\partial f^{ \pm}}{\partial T}+\frac{\partial^{3} f^{ \pm}}{\partial \xi_{ \pm}^{3}}+f^{ \pm} \frac{\partial f^{ \pm}}{\partial \xi_{ \pm}}\right)=\gamma f^{ \pm}
$$

We consider the initial-value problem for the uncoupled Ostrovsky equations (48) starting with the initial values $\left.f^{ \pm}\right|_{T=0}$ given by (18). By the local and global well-posedness theory for the Ostrovsky equation [16, 27, 40, 41], a unique global solution $f^{ \pm} \in C\left(\mathbb{R}_{+}, H_{\text {per }}^{s}(-L, L)\right)$ exists for any $\left.f^{ \pm}\right|_{T=0} \in H_{\text {per }}^{s}(-L, L)$ with $s>\frac{3}{4}$.

Remark 7. If $\gamma \neq 0$, solutions of the Ostrovsky equations (48) must satisfy the zero-mean constraints [2]. In our derivation, the zero-mean constraints are satisfied automatically because $U_{0}$ and $f^{ \pm}$are the zero-mean parts of the $(2 L)$-periodic functions. Note that the oscillating term $\langle U\rangle=c_{0} \cos (\omega t)$ does not contribute to the Ostrovsky equations (48). Consequently, as $\gamma \rightarrow 0$, the limiting $K d V$ equation (48) is different from the $K d V$ equation (24) if $c_{0} \neq 0$.

After the constraints (47) are substituted back into the differential equations (46), we obtain the linear inhomogeneous equations

$$
\begin{aligned}
\frac{d^{2} g_{n}}{d t^{2}}+\left(\frac{\pi n}{L}\right)^{2} g_{n}=-\frac{\pi^{2} n^{2}}{L^{2}} c_{0} \cos (\omega t)\left(a_{n}^{+} e^{\frac{i \pi n t}{L}}+a_{n}^{-} e^{-\frac{i \pi n t}{L}}\right) & \\
- & \frac{\pi^{2} n^{2}}{L^{2}} \sum_{k \in \mathbb{Z} \backslash\{0, n\}} a_{k}^{+} a_{n-k}^{-} e^{\frac{i \pi(2 k-n) t}{L}},
\end{aligned}
$$

subject to the initial conditions

$$
g_{n}(0)=0, \quad \partial_{t} g_{n}(0)=-\partial_{T} a_{n}^{+}(0)-\partial_{T} a_{n}^{-}(0) .
$$

This initial-value problem admits the following bounded solution:

$$
\begin{aligned}
g_{n}(t)= & \frac{c_{0} \pi^{2} n^{2}}{4 \pi^{2} n^{2}-\omega^{2} L^{2}}\left[\frac{2 \pi n i}{L} \frac{\sin (\omega t)}{\omega}\left(a_{n}^{+} e^{\frac{i \pi n t}{L}}-a_{n}^{-} e^{\frac{-i \pi n t}{L}}\right)-\cos (\omega t)\left(a_{n}^{+} e^{\frac{i \pi n t}{L}}+a_{n}^{-} e^{\frac{-i \pi n t}{L}}\right)\right] \\
& +\sum_{k \in \mathbb{Z} \backslash\{0, n\}} \frac{n^{2}}{4 k(k-n)} a_{k}^{+} a_{n-k}^{-} e^{\frac{i \pi(2 k-n) t}{L}}+G_{n} \cos \left(\frac{\pi n t}{L}\right)+H_{n} \sin \left(\frac{\pi n t}{L}\right),
\end{aligned}
$$

where $G_{n}$ and $H_{n}$ are constants of integrations to be found from the initial conditions for $g_{n}$.

Remark 8. Since $\omega=\sqrt{\epsilon \gamma}$, the first term in the explicit solution for $g_{n}$ grows in $t$ in the limit $\epsilon \rightarrow 0$ if $c_{0} \neq 0$ but it is nevertheless bounded by the $\mathcal{O}\left(\epsilon^{-1 / 2}\right)$ constant for any $\epsilon>0$. This fact implies that

$$
\left\|\epsilon U_{1}\right\|_{H_{\mathrm{per}}^{1}}=\mathcal{O}\left(c_{0} \epsilon^{1 / 2}\right) \quad \text { as } \quad \epsilon \rightarrow 0 .
$$


If the zero-mean velocity constraint $(12)$ is violated and $V_{0} \neq 0$, then the asymptotic procedure will give $\left\|\epsilon U_{1}\right\|_{H_{\mathrm{per}}^{1}}=\mathcal{O}(1)$ as $\epsilon \rightarrow 0$ and the first-order correction term (as well as all higherorder correction terms) become comparable with the leading-order approximation. This will clearly prevent us from justification of the long-wave approximation. This remark explains why we have set $V_{0}=0$ for $\gamma>0$ in the constraint (12).

Using the explicit solution for $g_{n}$, we rewrite the first-order correction term in the implicit form:

$$
U_{1}(x, t)=f_{c}(x, t)+f_{m}(x, t)+\phi^{-}\left(\xi_{-}, T\right)+\phi^{+}\left(\xi_{+}, T\right),
$$

where $f_{c}$ is given by $(26)$ and $(28), f_{m}$ is uniquely defined by

$$
\begin{aligned}
f_{m}(x, t)= & \frac{\sin (\omega t)}{\omega} \sum_{n \in \mathbb{Z} \backslash\{0\}} \frac{c_{0} \pi^{2} n^{2}}{4 \pi^{2} n^{2}-\omega^{2} L^{2}} \frac{2 \pi n i}{L}\left(a_{n}^{+}(T) e^{\frac{i \pi n t}{L}+\frac{i \pi n x}{L}}-a_{n}^{-}(T) e^{\frac{-i \pi n t}{L}+\frac{i \pi n x}{L}}\right) \\
& -\cos (\omega t) \sum_{n \in \mathbb{Z} \backslash\{0\}} \frac{c_{0} \pi^{2} n^{2}}{4 \pi^{2} n^{2}-\omega^{2} L^{2}}\left(a_{n}^{+}(T) e^{\frac{i \pi n t}{L}+\frac{i \pi n x}{L}}+a_{n}^{-}(T) e^{\frac{-i \pi n t}{L}+\frac{i \pi n x}{L}}\right),
\end{aligned}
$$

and functions $\phi^{ \pm}$are given by

$$
\phi^{ \pm}\left(\xi_{ \pm}, T\right)=\sum_{n \in \mathbb{Z}} b_{n}^{ \pm}(T) e^{\frac{i \pi n \xi_{ \pm}}{L}}
$$

subject to the initial conditions

$$
\begin{aligned}
\left.b_{n}^{ \pm}\right|_{T=0}= & \left.\frac{c_{0} \pi^{2} n^{2}}{4 \pi^{2} n^{2}-\omega^{2} L^{2}} a_{n}^{\mp}\right|_{T=0}-\left.\sum_{k \in \mathbb{Z} \backslash\{0, n\}} \frac{n(n \pm(2 k-n))}{8 k(k-n)}\left(a_{k}^{+} a_{n-k}^{-}\right)\right|_{T=0} \\
& \left.\mp \frac{L}{2 i \pi n}\left(\frac{d a_{n}^{+}}{d T}+\frac{d a_{n}^{-}}{d T}\right)\right|_{T=0} .
\end{aligned}
$$

Order $\mathcal{O}\left(\epsilon^{2}\right)$ : The second-order correction term satisfies the initial-value problem:

$$
\left\{\begin{array}{l}
\left(\partial_{t}^{2}-\partial_{x}^{2}\right) U_{2}=-2 \partial_{t T}^{2} U_{1}-\partial_{T}^{2} U_{0}+c_{0} \cos (\omega t) \partial_{x}^{2} U_{1}+\partial_{x}^{2}\left(U_{0} U_{1}\right) \\
\quad+\partial_{t t x x}^{4} U_{1}+2 \partial_{t T x x}^{4} U_{0}-\gamma U_{1}, \\
\left.U_{2}\right|_{t=0}=0, \\
\left.\partial_{t} U_{2}\right|_{t=0}=-\left.\partial_{T} U_{1}\right|_{t=0} .
\end{array}\right.
$$

Repeating the procedure of removing the resonant terms $e^{ \pm \frac{i \pi n t}{L}}$ and using again the fact that $\cos (\omega t)$ and $\sin (\omega t)$ do not produce the resonant terms, we define uniquely the evolution problem for the Fourier coefficients of the functions $\phi^{ \pm}$in 51 :

$$
\begin{array}{r}
-\frac{d^{2} a_{n}^{ \pm}}{d T^{2}} \mp \frac{2 \pi i n}{L} \frac{d b_{n}^{ \pm}}{d T}+\frac{\pi^{4} n^{4}}{L^{4}} b_{n}^{ \pm} \mp \frac{2 i \pi^{3} n^{3}}{L^{3}} \frac{d a_{n}^{ \pm}}{d T} \\
-\frac{\pi^{2} n^{2}}{2 L^{2}} \sum_{k \in \mathbb{Z} \backslash\{0, n\}} a_{k}^{ \pm} b_{n-k}^{ \pm}-\frac{\pi^{2} n^{2}}{L^{2}} \sum_{k \in \mathbb{Z} \backslash\{0, n\}} \frac{(n-k)^{2}}{4 n k} a_{n}^{ \pm}\left|a_{k}^{\mp}\right|^{2}-\gamma b_{n}^{ \pm}=0 .
\end{array}
$$


These equations are equivalent to the linearized Ostrovsky equations

$$
\frac{\partial}{\partial \xi_{ \pm}}\left(\mp 2 \frac{\partial \phi^{ \pm}}{\partial T}+\frac{\partial^{3} \phi^{ \pm}}{\partial \xi_{ \pm}^{3}}+\frac{\partial}{\partial \xi_{ \pm}} f^{ \pm} \phi^{ \pm}\right)=\gamma \phi^{ \pm}+\frac{\partial^{2} f^{ \pm}}{\partial T^{2}} \mp 2 \frac{\partial^{4} f^{ \pm}}{\partial \xi_{ \pm}^{3} T}+\frac{\partial^{2} f_{s}^{ \pm}}{\partial \xi_{ \pm}^{2}}
$$

with the same definition for $f_{s}^{ \pm}$as in $(33)$. The closed form expressions for the initial conditions in terms of the leading order solutions $f^{ \pm}$can be found in [18, 19].

After the constraints (54) are substituted back to the initial-value problem (53), we can obtain a bounded solution for $U_{2}(x, t)$. Note that the bounded solutions satisfies

$$
\left\|\epsilon^{2} U_{2}\right\|_{H_{\mathrm{per}}^{1}}=\mathcal{O}\left(c_{0} \epsilon\right) \quad \text { as } \quad \epsilon \rightarrow 0
$$

because of the oscillatory behavior of the functions $\cos (\omega t)$ and $\sin (\omega t)$ with $\omega=\sqrt{\epsilon \gamma}$. This completes the construction of the formal asymptotic expansion (15) up to and including the $\mathcal{O}\left(\epsilon^{2}\right)$ terms.

4.2. Justification. The following theorem gives the main result on the justification of the long-wave approximation.

Theorem 2. Assume that $(F, V) \in H_{\mathrm{per}}^{1}(-L, L) \times H_{\mathrm{per}}^{1}(-L, L)$ subject to the zero-mean constraint (12) on $V$. Fix $s \geq 10$ and let $f^{ \pm} \in C\left(\mathbb{R}, H_{\text {per }}^{s}(-L, L)\right)$ be global solutions of the Ostrovsky equations (48) starting with the initial conditions (18). Let $U_{0}$ and $U_{1}$ be given by (19) and (49) with (28), (50), (51), and (52). There is $\epsilon_{0}>0$ such that for all $\epsilon \in\left(0, \epsilon_{0}\right)$ and all $\epsilon$-independent $T_{0}>0$, there is $\epsilon$-independent constant $C>0$ such that for all $t_{0} \in\left[0, T_{0} / \epsilon\right]$, the local solution of the regularized Boussinesq equation (2) satisfies

$$
\sup _{t \in\left[0, t_{0}\right]}\left\|U-c_{0} \cos (\omega t)-U_{0}-\epsilon U_{1}\right\|_{H_{\mathrm{per}}^{1}} \leq C \epsilon t_{0}\left(c_{0}+\epsilon\right) .
$$

If, in addition, $\phi^{ \pm}$in (51), satisfies the linearized Ostrovsky equations (55) subject to the initial conditions (52) and $s$ is sufficiently large, then for all $\epsilon \in\left(0, \epsilon_{0}\right)$ and all $\epsilon$-independent $T_{0}>0$, there is an $\epsilon$-independent constant $C>0$ such that

$$
\sup _{t \in\left[0, T_{0} / \epsilon\right]}\left\|U-c_{0} \cos (\omega t)-U_{0}-\epsilon U_{1}\right\|_{H_{\mathrm{per}}^{1}} \leq C \epsilon\left(c_{0}+\epsilon\right) .
$$

Remark 9. The bounds (56) and (57) of Theorem 2 are larger then the bounds (34) and (35) of Theorem 1 if $c_{0} \neq 0$ but they still complete justification of the long-wave approximation up to the first-order correction term because $\left\|\epsilon U_{1}\right\|_{H_{\mathrm{per}}^{1}}=\mathcal{O}\left(c_{0} \epsilon^{1 / 2}\right)$ as $\epsilon \rightarrow 0$.

Remark 10. If $c_{0}=0$, then bounds (56) and (57) become bounds (34) and (35) because the periodic driving terms $\cos (\omega t)$ and $\sin (\omega t)$ are not present in all the expansions.

The proof of this justification theorem is similar to the proof of Theorem 1 after the energy of the error term for the formal asymptotic expansion as in (36) and (40) is modified to include the $\epsilon \gamma$-term as in the energy function (9). 


\section{Numerical iLlustrations}

5.1. Boussinesq equation with $\gamma=0$. Let us consider the initial-value problem for the regularized Boussinesq equation (13) starting with initial data

$$
\left\{\begin{array}{l}
\left.U\right|_{t=0}=3 k^{2} \operatorname{sech}^{2}\left(\frac{k x}{2}\right) \\
\left.U_{t}\right|_{t=0}=3 k^{3} \operatorname{sech}^{2}\left(\frac{k x}{2}\right) \tanh \left(\frac{k x}{2}\right)
\end{array}\right.
$$

where $k>0$ is an arbitrary parameter. The initial data is defined on the periodic domain $-L \leq x \leq L$ and the mean value is given by

$$
c_{0}=\left.\frac{1}{2 L} \int_{-L}^{L} U\right|_{t=0} d x=\frac{6 k}{L} \tanh \left(\frac{k L}{2}\right) .
$$

When $L \rightarrow \infty, c_{0} \rightarrow 0$, and the initial data (58) corresponds at the leading order to a solitary wave of the $\mathrm{KdV}$ equation (24) propagating to the right:

$$
f^{+}\left(\xi_{+}, T\right)=0, \quad f^{-}\left(\xi_{-}, T\right)=3 k^{2} \operatorname{sech}^{2}(z), \quad z=\frac{k}{2}\left(\xi_{-}-\frac{k^{2}}{2} T\right) .
$$

Combining (19) and (25), we obtain the weakly nonlinear solution in the form

$$
U=f^{-}\left(\xi_{-}, T\right)+\epsilon\left[\phi^{-}\left(\xi_{-}, T\right)+\phi^{+}\left(\xi_{+}, T\right)\right]+\mathcal{O}\left(\varepsilon^{2}\right),
$$

where the correction terms $\phi^{ \pm}$satisfy the linearized $\mathrm{KdV}$ equations

$$
-2 \partial_{T} \phi^{+}+\partial_{\xi_{+}}^{3} \phi^{+}=0
$$

and

$$
\partial_{\xi^{-}}\left(2 \partial_{T} \phi^{-}+\partial_{\xi_{-}}^{3} \phi^{-}+\partial_{\xi_{-}}\left(f^{-} \phi^{-}\right)\right)=\partial_{T}^{2} f^{-}+2 \partial_{T} \partial_{\xi_{-}}^{3} f^{-},
$$

subject to the initial data

$$
\left.\phi^{+}\right|_{T=0}=\frac{3 k^{4}}{4} \operatorname{sech}^{2}\left(\frac{k \xi_{+}}{2}\right),\left.\quad \phi^{-}\right|_{T=0}=-\frac{3 k^{4}}{4} \operatorname{sech}^{2}\left(\frac{k \xi_{-}}{2}\right) .
$$

In what follows, we consider simplification of all expressions in the case of solitary waves with sufficiently large $L$.

Because $\partial_{T} f^{-}=-\frac{k^{2}}{2} \partial_{\xi_{-}} f^{-}$, we can integrate the linearized $\mathrm{KdV}$ equation 62 in $\xi_{-}$, subject to the zero boundary conditions, and obtain:

$$
2 \partial_{T} \phi^{-}+\partial_{\xi_{-}}^{3} \phi^{-}+\partial_{\xi_{-}}\left(f^{-} \phi^{-}\right)=\partial_{\xi^{-}}\left(\frac{k^{4}}{4} f^{-}-k^{2} \partial_{\xi_{-}}^{2} f^{-}\right) .
$$

Now, we recall that $f^{-}$solves the stationary KdV equation, which implies that

$$
\left(k^{2}-\partial_{\xi_{-}}^{2}+f^{-}\right) \partial_{\xi_{-}} f^{-}=0 \text {. }
$$

Using the decomposition

$$
\phi^{-}\left(\xi_{-}, T\right)=a k^{4} T \partial_{\xi_{-}} f^{-}+\psi^{-}(z), \quad z=\frac{k \xi_{-}}{2},
$$

where $a$ is a constant to be defined, we integrate the linearised equation for $\psi^{-}(z)$, with zero boundary conditions, to obtain

$$
\left(4-\partial_{z}^{2}-12 \operatorname{sech}^{2}(z)\right) \psi^{-}=3 k^{4}\left((3+8 a) \operatorname{sech}^{2}(z)-6 \operatorname{sech}^{6}(z)\right) .
$$


This equation is solved with $\psi^{-}=3 k^{4} \operatorname{sech}^{2}(z)$ and $a=-\frac{3}{8}$. Therefore, the initial-value problem for the linearized inhomogeneous $\mathrm{KdV}$ equation 62 is solved by the function

$$
\phi^{-}\left(\xi_{-}, T\right)=\tilde{\phi}^{-}\left(\xi_{-}, T\right)-\frac{3}{8} k^{4} T \frac{\partial f^{-}}{\partial \xi_{-}}+k^{2} f^{-}
$$

where $\tilde{\phi}^{-}\left(\xi_{-}, T\right)$ is the solution to the Cauchy problem for the homogeneous linearized KdV equation:

$$
2 \partial_{T} \tilde{\phi}^{-}+\partial_{\xi_{-}}^{3} \tilde{\phi}^{-}+\partial_{\xi_{-}}\left(f^{-} \tilde{\phi}^{-}\right)=0
$$

starting with the initial data

$$
\left.\tilde{\phi}^{-}\right|_{T=0}=-\frac{15 k^{4}}{4} \operatorname{sech}^{2}\left(\frac{k \xi_{-}}{2}\right) .
$$

Note that the solutions of the linearised homogeneous KdV equations (61) and (64) disperse to zero, so the effect of nonzero initial data decays in time.

We now compare direct numerical simulations of the Boussinesq equation (13) with the weakly nonlinear solution 60 . We discretise the spatial domain into $N$ equally spaced points and solve the Boussinesq equation in Matlab using a pseudo-spectral method, based on the Fast Fourier Transform (FFT) [11]. The accuracy of the numerical method is far in excess of what is required for comparisons with the weakly nonlinear solution (60), nevertheless, extensions for further more accurate requirements are trivially achieved by further decreasing the time step and/or increasing the number of harmonics in the FFT. Similarly we find the higher order terms $\phi^{ \pm}$numerically using pseudo-spectral methods based on the FFT algorithm, analogous to the method used to solve the KdV equation in [40] .

To consider the error of the weakly nonlinear solution, under the initial data (58), we first introduce some notation. We define the numerical solution of the Boussinesq equation (13) as $U_{\text {num }}$ and the weakly nonlinear solutions as

$$
U_{1}=f^{-}, \quad U_{2}=f^{-}+\epsilon\left(\phi_{-}^{\text {num }}+\phi_{+}^{\text {num }}\right),
$$

where $\phi_{ \pm}^{\text {num }}$ are numerical solutions of the linearized KdV equations (61) and (62). When $\phi_{ \pm}$ are fixed at their initial condition $(63)$, we denote this solution as $U_{12}$. Note that the latter solution was previously studied in [18, 19].

Figure 1 presents numerical solutions for $k=\frac{1}{\sqrt{3}}$ and $\epsilon=0.1$. The top panels of Figure 1 depict the evolution of the numerical solution $U_{\text {num }}$ and the weakly nonlinear solutions $U_{1}$, $U_{12}$, and $U_{2}$ at times $t=1$ (left) and $t=1 / \epsilon$ (right). The middle panels of Figure 1 show the close-up of the area near the maximum of the right-propagating solitary wave at $t=1$ (left) and of the small left-propagating wave at $t=1 / \epsilon$ (right). Note that the leading order approximation $U_{1}$ does not capture the generation of this left-propagating wave at all. The bottom panels of Figure 1 also illustrate the error terms for each of the weakly nonlinear solutions relative to the numerical solution $U_{\text {num }}$, for a particular $\epsilon$. For the time interval considered, it is clear that there is a significant improvement in the error of the weakly nonlinear solutions $U_{12}$ and $U_{2}$ compared to the leading order approximation $U_{1}$.

The important question is now: how does the error of the solutions $U_{1}, U_{12}$ and $U_{2}$ scale with $\epsilon$ ? To analyse the error of the weakly nonlinear solutions in more detail, we consider 


$$
t=1
$$

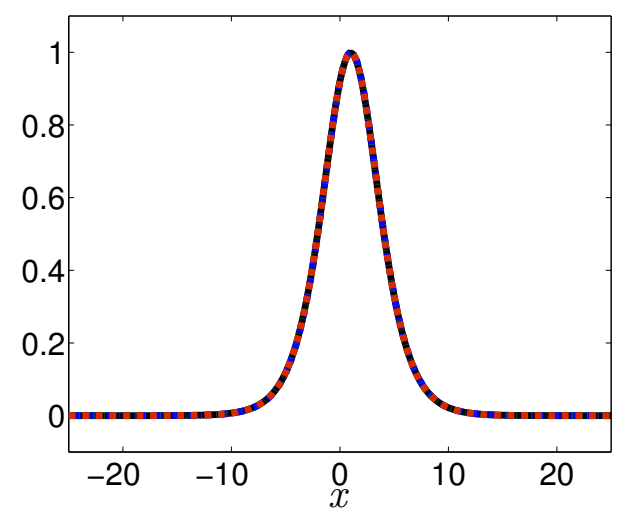

(a) $U_{\text {num }}(-) \quad U_{1}(-.-) \quad U_{12}(\cdots) \quad U_{2}(--)$

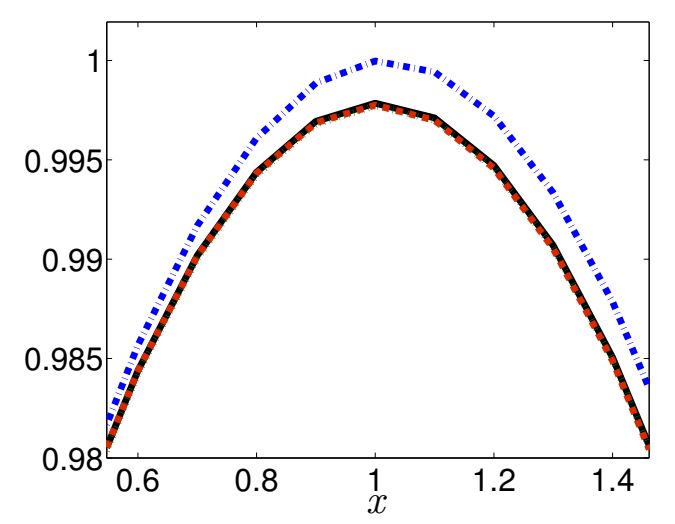

(c) $U_{\text {num }}(-) \quad U_{1}(-.-) \quad U_{12}(\cdots) \quad U_{2}(--)$

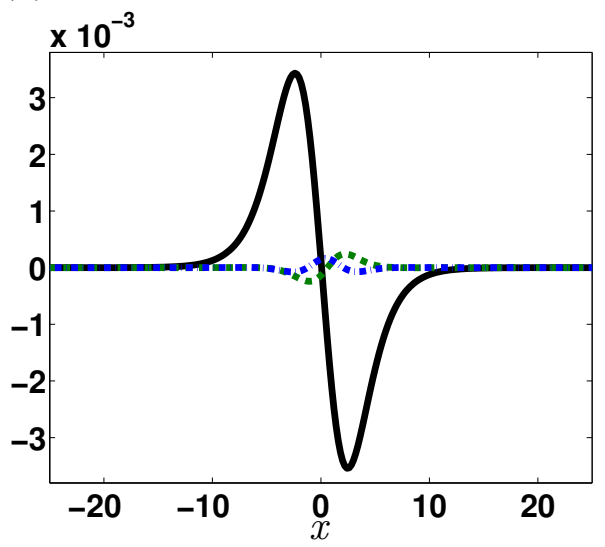

(e) $\left(U_{\text {num }}-U_{1}\right)(-)\left(U_{\text {num }}-U_{12}\right)(-.-)$ $\left(U_{\text {num }}-U_{2}\right)(--)$ $t=1 / \epsilon$

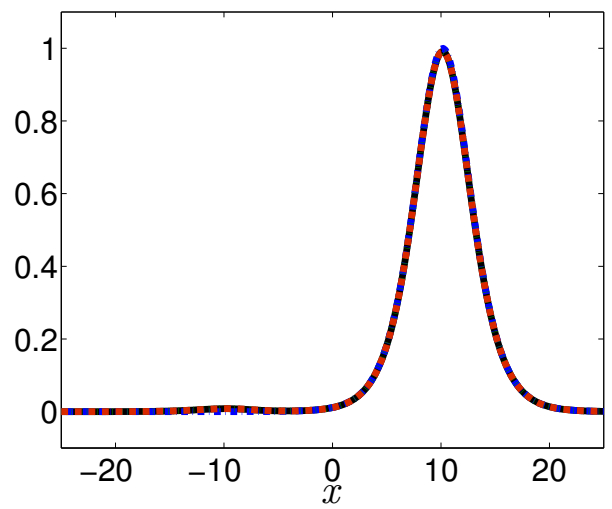

(b) $U_{\mathrm{num}}(-) \quad U_{1}(-.-) \quad U_{12}(\cdots) \quad U_{2}(--)$

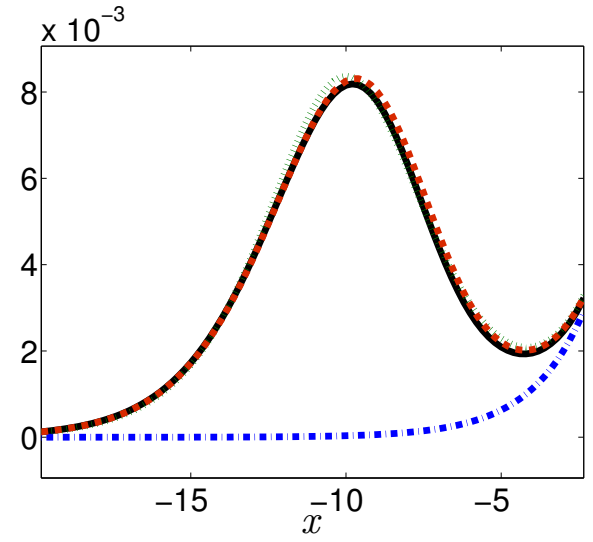

(d) $U_{\text {num }}(-) \quad U_{1}(-.-) \quad U_{12}(\cdots) \quad U_{2}(--)$

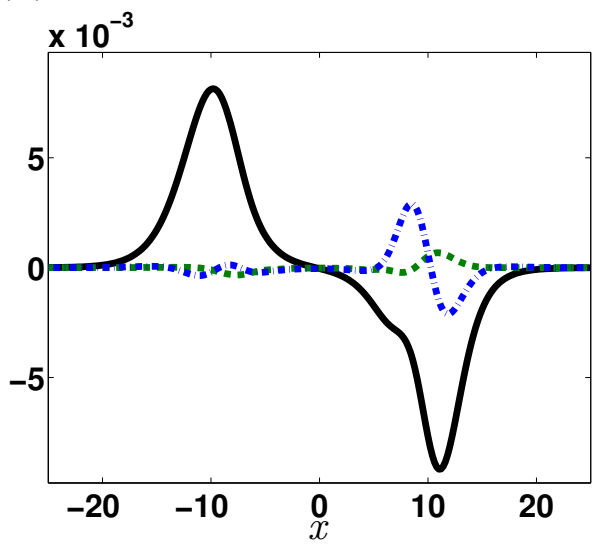

(f) $\left(U_{\text {num }}-U_{1}\right)(-)\left(U_{\text {num }}-U_{12}\right)(-.-)$ $\left(U_{\text {num }}-U_{2}\right)(--)$

Figure 1. Comparison of the weakly nonlinear solutions $U_{1}, U_{12}$, and $U_{2}$ with the numerical solution $U_{\text {num }}$ for $k=1 / \sqrt{3}, \epsilon=0.1, \gamma=0$ at (a) $t=1 \&$ (b) $t=1 / \epsilon$, with the close-up of some areas (c) \& (d) and the error plots (e) \& (f) at the respective times. Numerical parameters: $\Delta t=0.01, \Delta T=0.00125$ and $L=2000, N=2 \times 10^{4}$. 
the maximum absolute error over $x$ defined as

$$
e_{t}^{i}=\max _{-L \leq x \leq L}\left|U_{\text {num }}(x, t)-U_{i}(x, t)\right|, \quad i=1,12,2 .
$$

We use a least squares power fit to determine how the maximum absolute error of the weakly nonlinear solution at each order varies with the small parameter $\epsilon$. First we write the maximum errors defined in $(66)$ in the form

$$
e_{t}^{i}=C_{i} \epsilon^{\alpha_{i}}, \quad \text { for } \quad i=1,12,2,
$$

corresponding to each order of $\epsilon$ in the weakly nonlinear solution (65). Taking logs of the errors in this form and considering a range of $\epsilon$, one can find the coefficients $C_{i}$ and $\alpha_{i}$, with the latter revealing how the maximum absolute errors scale with $\epsilon$. We find the coefficients using Matlab's "polyfit" command.

$$
t=1
$$

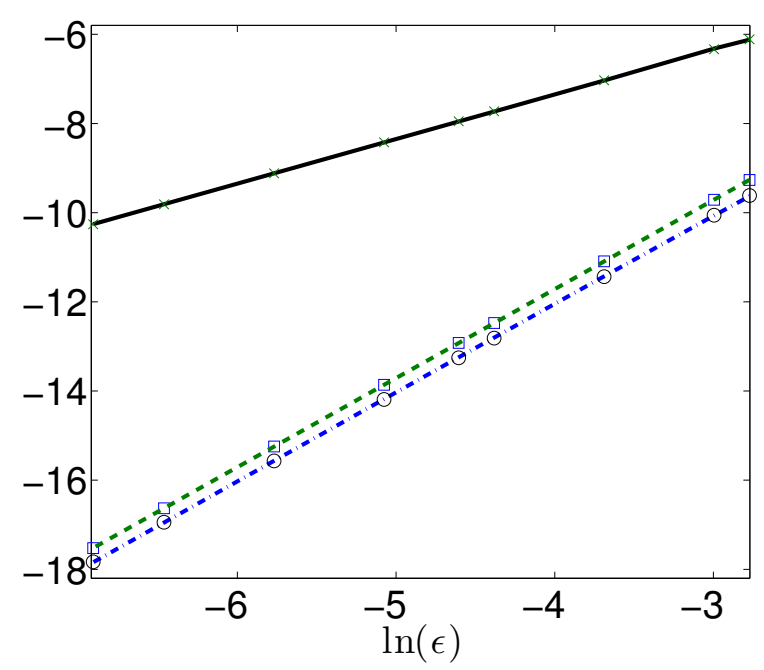

$$
\ln \left(e_{t}^{1}\right)(-) \quad \alpha_{1} \ln (\epsilon)+\ln \left(C_{1}\right)(\times \times)
$$$$
\ln \left(e_{t}^{2}\right)(--)
$$

$$
t=1 / \epsilon
$$

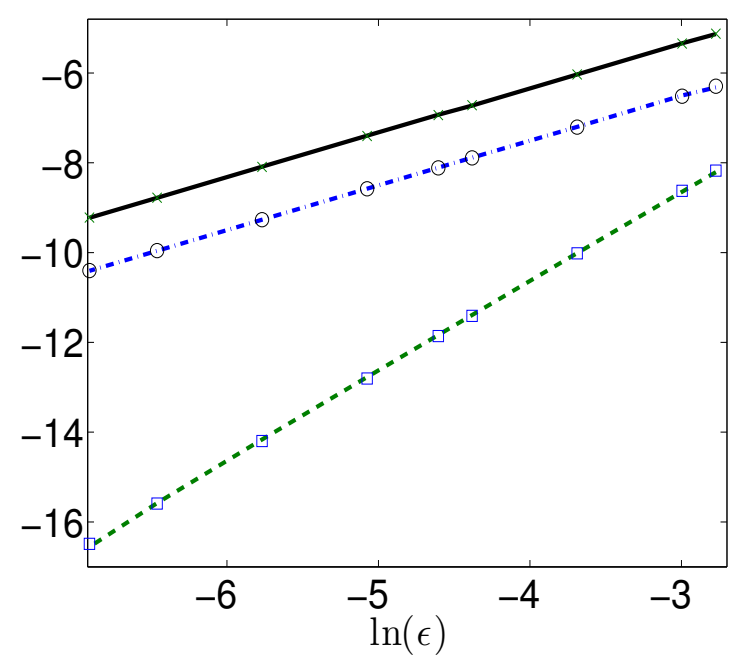

$\ln \left(e_{t}^{12}\right)(-.-) \quad \alpha_{12} \ln (\epsilon)+\ln \left(C_{12}\right)(\bigcirc \bigcirc)$ $\alpha_{2} \ln (\epsilon)+\ln \left(C_{2}\right)(\square \square)$

Figure 2. Double log plot of absolute errors versus $\epsilon$ at $t=1$ and $t=1 / \epsilon$. Numerical parameters are the same as in Figure 1. Coefficients $\alpha_{1,12,2}$ and $C_{1,12,2}$ are given in Table 1 .

In Figure 2 we display double log plots of the maximum absolute errors, which we find explicitly from simulations against $\epsilon$, and the log of the errors as defined in (67), both at times $t=1$ (left) and $t=1 / \epsilon$ (right). As depicted in Figure 2, we find the weakly nonlinear solution $U_{2}$ dramatically improves the scaling of the maximum absolute error at the time $t=1 / \epsilon$, in comparison with the solution $U_{12}$. Furthermore, the maximum absolute error of $U_{2}$ scales almost precisely as $\mathcal{O}\left(\epsilon^{2}\right)$, in agreement with the rigorous error estimates of Theorem 1 ,

We note from Figure 2 the strong similarity in the error scalings of the leading order solution $U_{1}$ with the higher order solution $U_{12}$ at $t=1 / \varepsilon$. This was not observed in [18, 19] 
since the detailed analysis of the error term was not undertaken. However, it must be noted, upon direct comparison of the two solutions, that the maximum absolute error of the solution $U_{12}$ is still significantly smaller than the error of the solution $U_{1}$. This is because the constant $C_{12}$ in the error term (67) is substantially smaller than the corresponding leading order constant $C_{1}$ (see Table 1 ).

\begin{tabular}{c|cccc}
\hline & $\gamma=0$ & \multicolumn{2}{c}{$\gamma>0$} \\
\hline & $t=1$ & $t=1 / \epsilon$ & $t=1$ & $t=1 / \epsilon$ \\
\hline$\alpha_{1}$ & 1.0032 & 0.9906 & 1.0026 & 0.9690 \\
$\alpha_{12}$ & 1.9870 & 0.9928 & - & - \\
$\alpha_{2}$ & 1.9970 & 2.0103 & 1.9302 & 1.9515 \\
$C_{1}$ & 0.03576 & 0.04979 & 0.1668 & 0.4851 \\
$C_{12}$ & 0.01648 & 0.02896 & - & - \\
$C_{2}$ & 0.02407 & 0.07417 & 0.5474 & 9.2062 \\
\hline
\end{tabular}

TABLE 1. Maximum absolute error scaling parameters corresponding to results illustrated in Figures 2 \& 4 .

5.2. Boussinesq equation with $\gamma>0$. Let us now consider an initial-value problem for the regularized Boussinesq equation (2) starting with initial data

$$
\left\{\begin{aligned}
\left.U\right|_{t=0} & =3 k^{2} \operatorname{sech}^{2}\left(\frac{k x}{2}\right)-\hat{\alpha}\left[\operatorname{sech}^{2}\left(\frac{k\left(x+x_{0}\right)}{2}\right)+\operatorname{sech}^{2}\left(\frac{k\left(x-x_{0}\right)}{2}\right)\right], \\
\left.U_{t}\right|_{t=0} & =3 k^{3} \operatorname{sech}^{2}\left(\frac{k x}{2}\right) \tanh \left(\frac{k x}{2}\right)-k \hat{\alpha}\left[\operatorname{sech}^{2}\left(\frac{k\left(x+x_{0}\right)}{2}\right) \tanh \left(\frac{k\left(x+x_{0}\right)}{2}\right)\right. \\
& \left.+\operatorname{sech}^{2}\left(\frac{k\left(x-x_{0}\right)}{2}\right) \tanh \left(\frac{k\left(x-x_{0}\right)}{2}\right)\right],
\end{aligned}\right.
$$

where $x_{0}$ is an arbitrary shift along $x$. The initial data is defined on the periodic domain $-L \leq x \leq L$. If we choose $\hat{\alpha}$ to be

$$
\hat{\alpha}=\frac{3 k^{2} \tanh (k L / 2)}{\tanh \left(k\left(L+x_{0}\right) / 2\right)+\tanh \left(k\left(L-x_{0}\right) / 2\right)},
$$

then the mean value of $\left.U\right|_{t=0}$ is zero, $c_{0}=0$.

The weakly nonlinear solution is still given by the expansion (60) but the leading order term $f^{-}$is now a solution to the Ostrovsky equation (48) starting with the initial data

$$
\left.f^{-}\right|_{T=0}=3 k^{2} \operatorname{sech}^{2}\left(\frac{k \xi^{-}}{2}\right)-\hat{\alpha}\left[\operatorname{sech}^{2}\left(\frac{k\left(\xi^{-}+x_{0}\right)}{2}\right)+\operatorname{sech}^{2}\left(\frac{k\left(\xi^{-}-x_{0}\right)}{2}\right)\right] .
$$

The higher order terms $\phi^{ \pm}$satisfy the linearized Ostrovsky equations

$$
\partial_{\xi_{+}} \phi^{+}\left(-2 \partial_{T} \phi^{+}+\partial_{\xi_{+}}^{3} \phi^{+}\right)=\gamma \phi^{+}
$$

and

$$
\partial_{\xi^{-}}\left(2 \partial_{T} \phi^{-}+\partial_{\xi_{-}}^{3} \phi^{-}+\partial_{\xi_{-}}\left(f^{-} \phi^{-}\right)\right)=\gamma \phi^{-}+\partial_{T}^{2} f^{-}+2 \partial_{T} \partial_{\xi_{-}}^{3} f^{-},
$$

starting with the initial data

$$
\left\{\begin{array}{l}
\left.\phi^{+}\right|_{T=0}=-\Phi\left(\xi_{+}\right), \\
\left.\phi^{-}\right|_{T=0}=\Phi\left(\xi_{-}\right),
\end{array}\right.
$$


where $\Phi$ can be expressed in terms of the leading order solution $f^{-}$as

$$
\Phi(x)=\left.\frac{1}{2}\left(\int_{-L}^{x} f_{T}^{-}(s) d s-\frac{1}{2 L} \int_{-L}^{L}\left(\int_{-L}^{y} f_{T}^{-}(s) d s\right) d y\right)\right|_{T=0} .
$$

The function $f^{-}$is a solution to the Ostrovsky equation (48), and therefore the derivative $f_{T}^{-}$in 73 can be readily expressed as

$$
f_{T}^{-}=\frac{1}{2}\left(-f^{-} \partial_{\xi^{-}} f^{-}-\partial_{\xi^{-}}^{3} f^{-}+\gamma \partial_{\xi^{-}}^{-1} f^{-}\right) .
$$

Then, the formula $(73)$ shows, in particular, that the magnitude of the higher order corrections will be smaller if the initial data for the function $f^{-}$is localised, and for smaller values of the constant $\gamma$. To simplify our numerical simulation, we choose the initial data accordingly.

The Cauchy problems for equations (48), (70), and (71) are solved numerically and simultaneously at each time step. The Ostrovsky equation (48) can be solved numerically using both pseudo-spectral and finite difference methods (e.g. [14, 28, 30, 45]). We extend the spectral method used to solve the $\mathrm{KdV}$ equation in [39] in order to solve the linearized Ostrovsky equation (70) and the forced linearized Ostrovsky equation (71). The method used to solve the Boussinesq equation is an extension to the numerical method in [11.

We now compare direct numerical simulations of the Cauchy problem for the Boussinesq equation (2) with the weakly nonlinear solutions $U_{1}$ and $U_{2}$ defined by (65), as well as analyze the approximation error from $(\sqrt{66})$ and $(67)$.

Figure 3 presents numerical solutions for $k=\frac{1}{\sqrt{3}}, \epsilon=0.025$, and $\gamma=0.1$. The top panels of Figure 3 depict the evolution of the numerical solution $U_{\text {num }}$ and the weakly nonlinear solutions $U_{1}$ and $U_{2}$ at times $t=1$ (left) and $t=1 / \epsilon$ (right). The middle panels of Figure 3 show the close-up of the area near the maximum of the right-propagating wave at $t=1$ (left) and of the small left-propagating wave at $t=1 / \epsilon$ (right). We again note, similarly to our first example, that the leading order approximation $U_{1}$ does not capture the generation of this left-propagating wave at all.

We also note the important qualitative change in the dynamics of the solution, compared to the Boussinesq equation with $\gamma=0$ : the initial data generates the right-propagating nonlinear wave packet instead of a solitary wave. This dynamics agrees with the well known behaviour of solutions of the Ostrovsky equation [14, 15, where initial solitary-like disturbances lead to the formation of unsteady wave packets. The initial condition used in our example is closer to this wave packet than the pure solitary-like disturbance, and it has zero mass, which explains why there is less radiation.

The bottom panels of Figure 3 illustrate the evolution of errors for each of the weakly nonlinear solutions $U_{1}$ and $U_{2}$ relative to the numerical solution $U_{\text {num }}$, for a particular $\epsilon$. One can notice a distinct improvement in the accuracy of the weakly nonlinear solution $U_{2}$ compared with $U_{1}$.

Figure 4 displays double log plots of the maximum absolute error found explicitly from simulations and the log of the absolute maximum error as defined by (67). As one can see, the leading order maximum absolute error scales as $\mathcal{O}(\epsilon)$ for $U_{1}$ and as $\mathcal{O}\left(\epsilon^{2}\right)$ for $U_{2}$. This scaling agrees with the error estimates in Theorem 2. It must be noted, however, that the 


$$
t=1
$$

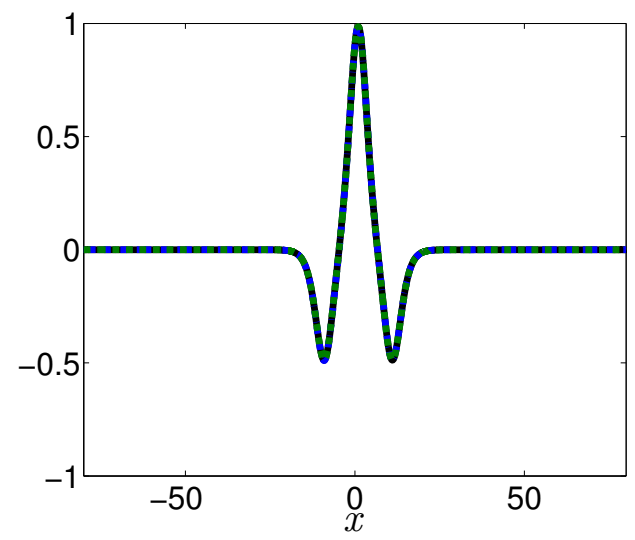

(a) $U_{\text {num }}(-) U_{1}(-.-) U_{2}(--)$

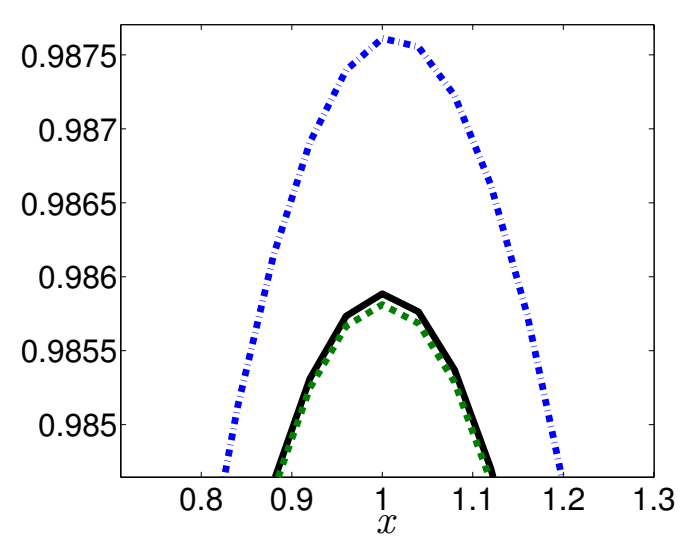

(c) $U_{\text {num }}(-) \quad U_{1}(-.-) \quad U_{2}(--)$

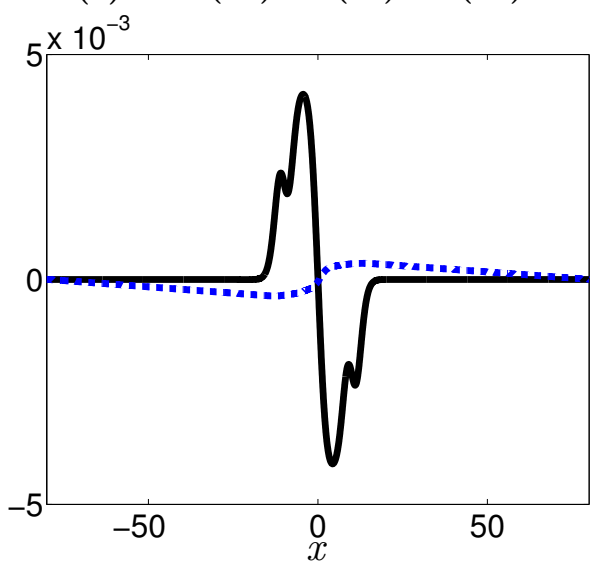

(e) $\left(U_{\text {num }}-U_{1}\right)(-)\left(U_{\text {num }}-U_{2}\right)(--)$ $t=1 / \epsilon$

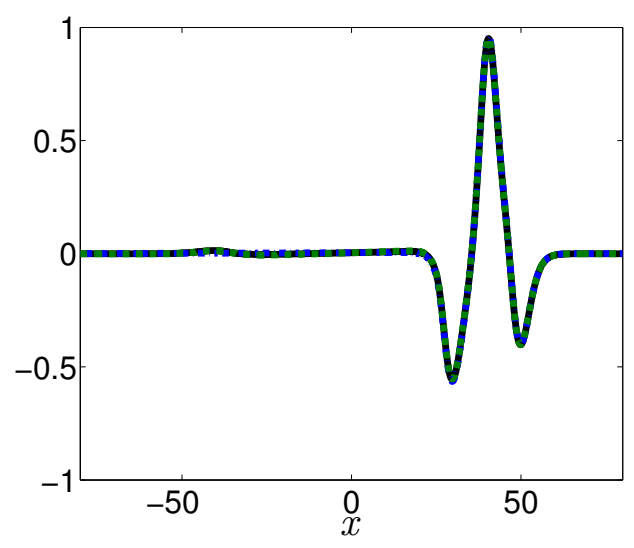

(b) $U_{\text {num }}(-) \quad U_{1}(-.-) \quad U_{2}(--)$

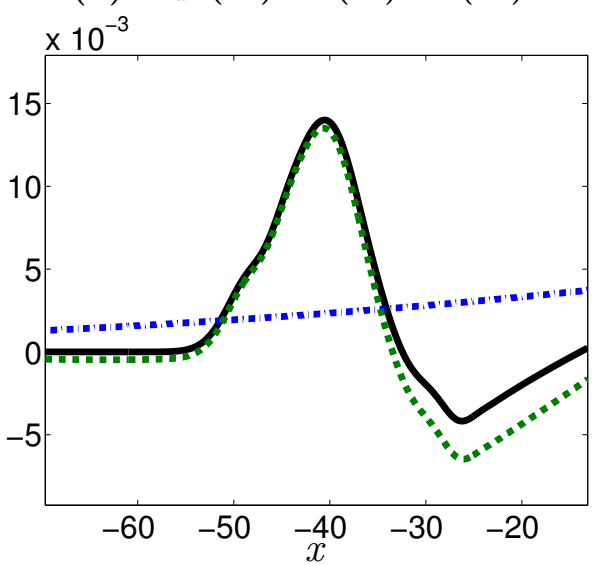

(d) $U_{\text {num }}(-) \quad U_{1}(-\cdot-) \quad U_{2}(--)$

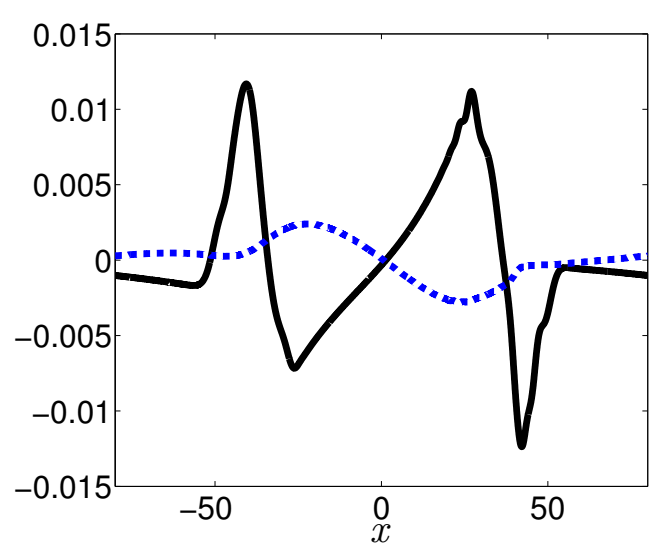

(f) $\left(U_{\text {num }}-U_{1}\right)(-)\left(U_{\text {num }}-U_{2}\right)(--)$

FIGURE 3. Comparison of the weakly nonlinear solutions $U_{1}$ and $U_{2}$ with the numerical solution $U_{\text {num }}$ for $k=1 / \sqrt{3}, \epsilon=0.025, \gamma=0.1$ at (a) $t=1 \&$ (b) $t=1 / \epsilon$, with the close-up of some areas (c) \& (d) and the error plots (e) \& (f) at the respective times. Numerical parameters: $\Delta t=0.01, \Delta T=0.000125$ and $L=80, N=4 \times 10^{4}$. 
constant $C_{2}$ of the higher-order error term $(67)$ is substantially larger than the corresponding leading order error term constant $C_{1}$, and both constants are larger than in the case of the Boussinesq equation (see Table 1). Therefore, although the error term scales as $\mathcal{O}\left(\varepsilon^{2}\right)$, as expected, it is substantially larger than in the case of $\gamma=0$ (compare the figures 1 (f) and 3 (f) and note the difference in the values of $\epsilon$ ).

$$
t=1
$$

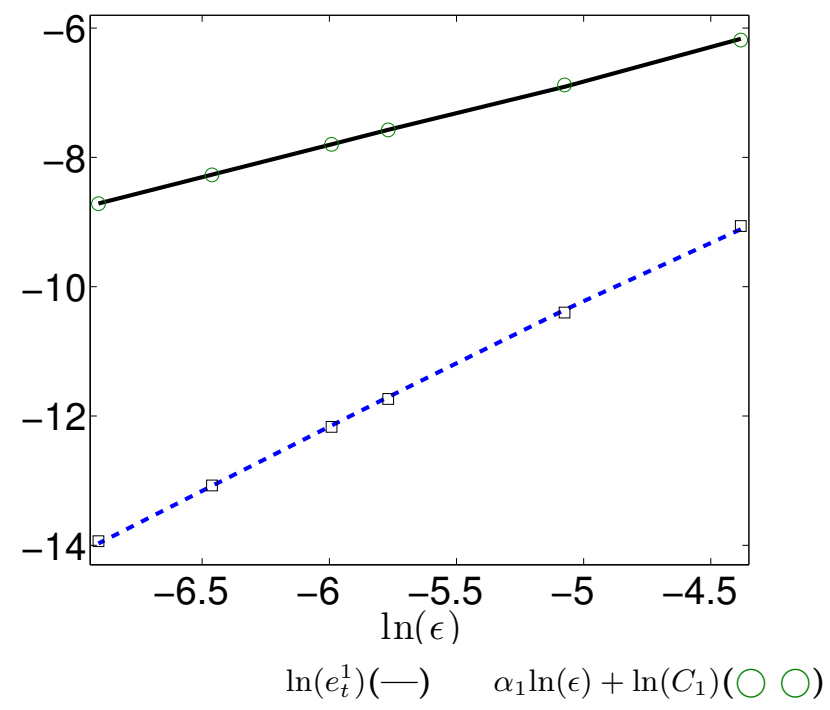

$t=1 / \epsilon$

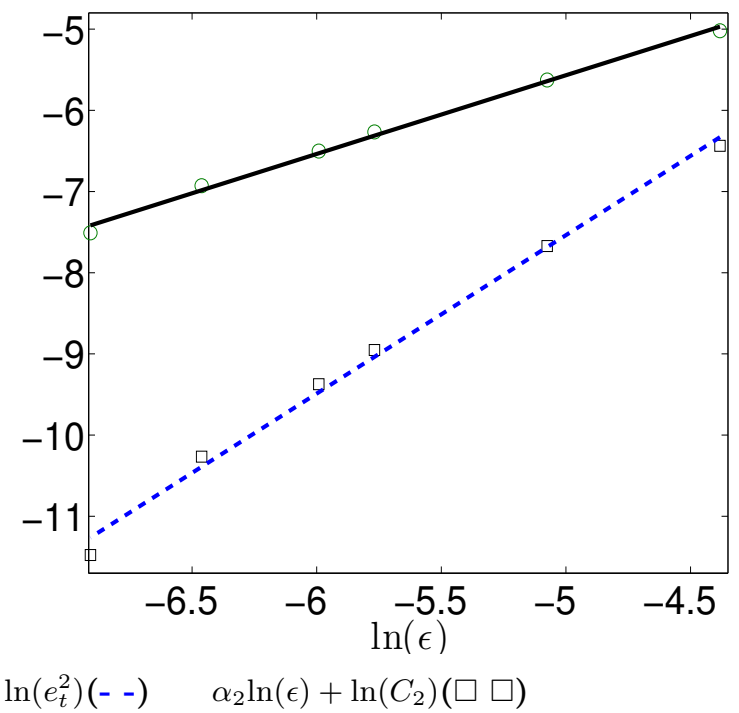

Figure 4. Double $\log$ plot of absolute errors versus $\epsilon$ at $t=1$ and $t=1 / \epsilon$. Numerical parameters are the same as in Figure 3. Coefficients $\alpha_{1,2}$ and $C_{1,2}$ are given in Table 1 .

\section{Conclusions}

In this paper we constructed a weakly nonlinear solution of the Cauchy problem for the regularized Boussinesq equation (1) with $\delta>0$ in the periodic domain. The weakly nonlinear solution is constructed in terms of solutions of two uncoupled Ostrovsky equations, extending the results obtained in [18, 19]. In our present paper, it was shown how the accuracy of the weakly nonlinear solution can be improved by using the linearized Ostrovsky equations for the two functions present in the first-order correction term. Although our consideration exceeded the accuracy of the physical problem formulation because the second-order correction terms were not included in the original Boussiness-type equation (1), the methodology can be generalized and extended to the case when the main equation includes these higher order terms.

Analytical results have been illustrated numerically, for the regularized Boussinesq equation with $\delta=0$ and $\delta>0$ in the large domain. The behavior of the error terms has been studied in details and the numerical results have shown excellent agreement with the analytical predictions. 
Acknowledgement: D.P. appreciates support and hospitality of the Department of Mathematical Sciences of Loughborough University. The research was supported by the LMS Scheme 2 grant and by the Loughborough University School of Science small grant. K.K. and K.M. thank C. Klein and D. Tseluiko for helpful discussions of the numerical methods.

\section{REFERENCES}

[1] W. Ben Youssef and T. Colin, "Rigorous derivation of Korteweg-de Vries-type systems from a general class of nonlinear hyperbolic systems", Math. Model. Numer. Anal. 34 (2000), 873-911.

[2] E.S. Benilov, "On the surface waves in a shallow channel with an uneven bottom", Stud. Appl. Math. 87 (1992), 1-14.

[3] F. Bethuel, P. Gravejat, J.-C. Saut and D. Smets, "On the Korteweg-de Vries long-wave approximation of the Gross-Pitaevskii equation I", Int. Math. Res. Notices 2009 (2009), 2700-2748.

[4] F. Bethuel, P. Gravejat, J.-C. Saut and D. Smets, "On the Korteweg-de Vries long-wave approximation of the Gross-Pitaevskii equation II", Comm. PDEs 35 (2010), 113-164.

[5] J.L. Bona, M. Chen, and J.C. Saut, "Boussinesq equations and other systems for small-amplitude long waves in nonlinear dispersive media. I. Derivation and linear theory", J. Nonlin. Sci. 12 (2002), 283-318.

[6] J.L. Bona, M. Chen, and J.C. Saut, "Boussinesq equations and other systems for small-amplitude long waves in nonlinear dispersive media. II. Nonlinear theory", Nonlinearity 17 (2004), 925-952.

[7] J.L. Bona, T. Colin, and D. Lannes, "Long wave approximations for water waves", Arch. Rational Mech. Anal. 178 (2005), 373-410.

[8] T. Cazenave and A. Haraux, An introduction to semilinear evolution equations (Oxford University Press, Oxford, 1998).

[9] J. Colliander, M. Keel, G. Staffilani, H. Takaoka and T. Tao, "Sharp global well-posedness for KdV and modified KdV on $\mathbb{R}$ and $\mathbb{T}$ ", J. Amer. Math. Soc. 16 (2003), 705-749.

[10] W. Craig, "An existence theory for water waves and the Boussinesq and Korteweg-de Vries scaling limits", Comm. PDEs 10 (1985), 787-1003.

[11] J. Engelbrecht, A. Salupere, K. Tamm, "Waves in microstructured solids and the Boussinesq paradigm", Wave Motion 48 (2011) 717-726.

[12] C. Fochesato, F. Dias, R. Grimshaw, "Generalized solitary waves and fronts in coupled Korteweg - de Vries systems", Physica D 210 (2005) 96 - 117.

[13] T. Gerkema, "A unified model for the generation and fission of internal tides in a rotating ocean", J. Marine Research 54 (1996), 421-450.

[14] R. Grimshaw, K. Helfrich, "Long-time solutions of the Ostrovsky equation", Stud. Appl. Math. 121 (2008) 71-88.

[15] R. Grimshaw, K. Helfrich, "The effect of rotation on internal solitary waves", IMA J. Appl. Math. 77 (2012) 326-339.

[16] G. Gui and Y. Liu, "On the Cauchy problem for the Ostrovsky equation with positive dispersion", Comm. Part. Diff. Eqs. 32 (2007), 1895-1916.

[17] Y. Hiraoka and Y. Kodama, "Normal form and solitons" in Integrability, Lecture Notes in Phys. $\mathbf{7 6 7}$ (Springer, Berlin, 2009), 175-214.

[18] K.R. Khusnutdinova and K.R. Moore, "Initial-value problem for coupled Boussinesq equations and a hierarchy of Ostrovsky equations", Wave Motion 48 (2011), 738-752.

[19] K.R. Khusnutdinova and K.R. Moore, "Weakly nonlinear extension of d'Alembert's formula", IMA J. Appl. Math. 77 (2012), 361-381.

[20] K.R. Khusnutdinova and A.M. Samsonov, "Fission of a longitudinal strain solitary wave in a delaminated bar", Phys. Rev. E 77 (2008), 066603 (14 pages).

[21] K.R. Khusnutdinova, A.M. Samsonov, and A.S. Zakharov, "Nonlinear layered lattice model and generalized solitary waves in imperfectly bonded structures", Phys. Rev. E 79 (2009), 056606 (14 pages). 
[22] Y. Kodama and A.V. Mikhailov, "Obstacles to asymptotic integrability" in Algebraic aspects of integrable systems, Progr. Nonlin. Differ. Eqs. Appl. 26 (Birkhäuser Boston, Boston, MA, 1997), 173-204.

[23] R. A. Kraenkel, M.A. Manna, and J.G. Pereira, "The Korteweg-De Vries hierarchy and long waterwaves", J. Math. Phys. 36 (1995), 307-320.

[24] R. A. Kraenkel, M.A. Manna, J.C. Montero, and J.G. Pereira, "Boussinesq solitary-wave as a multipletime solution of the Korteweg-De Vries hierarchy", J. Math. Phys. 36 (1995), 6822-6828.

[25] R. A. Kraenkel, M.A. Manna, J.C. Montero, and J.G. Pereira, "The role of the Korteweg-De Vries hierarchy in the $\mathrm{N}$-Soliton dynamics of the shallow water wave equation", J. Phys. Soc. Japan 66 (1997), 1277-1281.

[26] D. Lannes, "Secular growth estimates for hyperbolic systems", J. Diff. Eqs. 190 (2003), 466-503.

[27] F. Linares and A. Milanes, "Local and global well-posedness for the Ostrovsky equation", J. Diff. Eqs. 222 (2006), 325-340.

[28] Y. Miyatake, T. Yaguchi, T. Matsuo, "Numerical integration of the Ostrovsky equation based on its geometric structures", J. Comp. Phys. 231 (2012) 4542-4559.

[29] L.A. Ostrovsky, "Nonlinear internal waves in a rotating ocean", Oceanology 18 (1978), 119-125.

[30] M. Obregon and Y. Stepanyants, "On numerical solution of the Gardner - Ostrovsky equation", Math. Model. Nat. Phenom. 7 (2012) 113 - 130.

[31] D.E. Pelinovsky and D. Ponomarev, "Justification of a nonlinear Schrödinger model for laser beams in photopolymers", ZAMP, accepted (2013).

[32] D.E. Pelinovsky and G. Schneider, "Rigorous justification of the short-pulse equation", Nonlin. Diff. Eqs. Applicat. 20 (2013), 1277-1294.

[33] D.E. Pelinovsky, G. Simpson, and M.I. Weinstein, "Broad band solitons in a periodic and nonlinear Maxwell system", SIAM J. Appl. Dynam. Syst. 11 (2012), 478-506.

[34] A.V. Porubov, Amplification of nonlinear strain waves in solids (World Scientific, Singapore, 2003).

[35] A.M. Samsonov, Strain solitons in solids and how to construct them (Chapman and Hall/CRC, Boca Raton, 2001).

[36] G. Schneider, "The long wave limit for a Boussinesq equation", SIAM J. Appl. Math. 58 (1998), 12371245.

[37] G. Schneider and C.E. Wayne, "The long wave limit for the water wave problem I The case of zero surface tension", Comm. Pure Appl. Math. 53 (2000), 1475-1535.

[38] G. Schneider and C.E. Wayne, "The rigorous approximation of long-wavelength capillary-gravity waves", Arch. Rational Mech. Anal. 162 (2002), 247-285.

[39] L.N. Trefethen, "Spectral Methods in MATLAB", SIAM, Philadelphia, PA 19104-2688, 2000.

[40] K. Tsugawa, "Well-posedness and weak rotation limit for the Ostrovsky equation", J. Diff. Eqs. 247 (2009), 3163-3180.

[41] V. Varlamov and Y. Liu, "Cauchy problem for the Ostrovsky equation", Discr. Cont. Dyn. Syst. 10 (2004), 731-753.

[42] C.E. Wayne and J.D. Wright, "Higher order modulation equations for a Boussinesq equation", SIAM J. Appl. Dyn. Syst. 1 (2002), 271-302.

[43] J.D. Wright, "Corrections to the KdV approximation for water waves", SIAM J. Math. Anal. 37 (2006), 1161-1206.

[44] D. Yagi and T. Kawahara, "Strongly nonlinear envelope soliton in a lattice model for periodic structure", Wave Motion 34 (2001), 97-107.

[45] T. Yaguchi, T. Matsuo, M. Sugihara, "Conservative numerical schemes for the Ostrovsky equation", J. Comp. Appl. Math. 234 (2010) 1036-1048.

${ }^{1}$ Department of Mathematical Sciences, Loughborough University, Loughborough, Le11 3TU, UK, ${ }^{2}$ Department of Mathematics and Statistics, McMaster University, Hamilton ON, Canada, L8S 4K1 